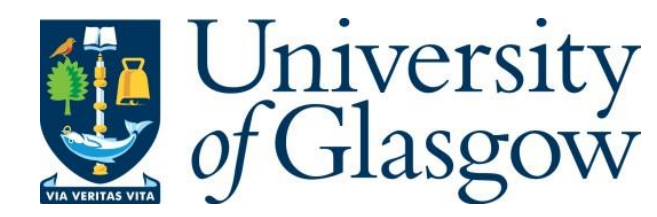

Ramírez-Torres, A., Di Stefano, S. and Grillo, A. (2021) Influence of non-local diffusion in avascular tumour growth. Mathematics and Mechanics of Solids, (doi:

10.1177/1081286520975086).

There may be differences between this version and the published version. You are advised to consult the publisher's version if you wish to cite from it.

http://eprints.gla.ac.uk/232136/

Deposited on: 18 March 2021

Enlighten - Research publications by members of the University of Glasgow http://eprints.gla.ac.uk 


\title{
Influence of non-local diffusion in avascular tumour growth
}

\author{
Ariel Ramírez-Torres ${ }^{1,2}$, Salvatore Di Stefano ${ }^{1}$, and Alfio Grillo ${ }^{1}$ \\ ${ }^{1}$ Dipartimento di Scienze Matematiche "G. L. Lagrange" Politecnico di \\ Torino, 10129. Torino, Italia \\ ${ }^{2}$ School of Mathematics and Statistics, Mathematics and Statistics \\ Building, University of Glasgow, University Place, Glasgow G128QQ, UK
}

\begin{abstract}
The availability and evolution of chemical agents play an important role in the growth of a tumour and, therefore, the mathematical description of their consumption is of special interest. Usually, Fick's law of diffusion is adopted for describing the local character of the evolution of chemicals. However, in a highly complex, heterogeneous medium, as is a tumour, the progression of chemical species could be influenced by non-local interactions. In this respect, our goal is to investigate the influence of such type of diffusion on the growth of a tumour in avascular stage. For our purposes, we consider a diffusion equation for the evolution of the chemical agents that accounts for the existence of non-local interactions in a non-Fickean manner, and that involves notions of Fractional Calculus. In particular, the introduction of derivatives or integrals of fractional type of order $\alpha \in \mathbb{R}$ has proven to be an effective mathematical tool in the description of various non-local phenomena. To achieve our goals, we adopt part of the modelling assumptions outlined in previous works of the authors, in which the growth of a tumour is described in terms of mass transfer among the tumour's constituents and structural changes that occur in the tumour itself in response to growth. The latter ones are characterised by means of the Bilby-Kröner-Lee decomposition of the deformation gradient tensor. We perform numerical simulations, whose results indicate the relevance of embracing a fractional framework in modelling tumour growth. Specifically, the real parameter $\alpha$ "dominates" the way in which the tumour grows, since it permits to model a variety of growth patterns ranging from the standard growth to no growth at all.
\end{abstract}

Keywords Tumour growth, non-Fickean diffusion, non-local interactions, inelastic distortions 


\section{Introduction}

For several years now, the scientific literature has experienced an important increase in the mathematical modelling of tumour growth (see e.g. [20, 14, 8, 64, 100, 78, 17, 107, 66, 97, 65] and the references therein). However, there is still the necessity for understanding the connections among the different processes of chemical, biological and/or mechanical nature that take place at different time and length scales and influence the evolution of a tumour.

From the mechanical perspective, the growth of a tumour is closely related to the appearance of transformations of its internal structure that arise in response to mass changes, which may be driven by its chemo-mechanical environment and coexist with the visible deformation of the tumour itself [39, 32, 95]. A relevant aspect of this phenomenology is that the structural transformations are often accompanied by the production of residual stresses [98, 173, 52, 28, 101. In this respect, we mention the series of experiments conducted by Stylianopoulos et al. 110 on tumour spheroids, which indicate the existence of an incompatible, stress-free state for such systems and, thus, suggest to interpret growth in terms of inelastic distortions in addition to mere changes of shape. This conclusion permits to invoke the Bilby-Kröner-Lee (BKL) multiplicative decomposition of the deformation gradient tensor [85, 52, 102. As long as volumetric growth is concerned and, as in the case of the present work, no other types of structural transformations are accounted for, the BKL decomposition reduces to decomposing the deformation gradient tensor into two contributions. One is related to the changes of the tissue's internal structure due to the gain or loss of mass, and the other one to distortions of purely elastic nature (note that, here and in the sequel, we shall use the terms "tumour" and "tissue" interchangeably). We refer to the works [102, 152, 94, 27, 101, 56, and to the references therein, for a more complete discussion on the BKL multiplicative decomposition.

It is worth noting that, although the inelastic distortions accompanying growth play an important role on its evolution 61, 6, 4, 151, 80, which may also be partially self-driven [41, 101, it is clear that the growth of a tumour is strongly conditioned by the presence of chemical agents of various nature, such as nutrients. Therefore, in order to elaborate a model of tumour growth, it is crucial to be able to model the evolution of chemical substances. Fick's law of diffusion is largely adopted for this purpose, even though it has often turned out to be inconsistent with the results of some observed transport processes [48, 21, 31, which are thus referred to as non-Fickean. In fact, non-Fickean diffusion processes have been recognised in several biological tissues, including cells [48, 31], neuromuscular junctions 74 and brain tissue [21, among others. In particular, the experiments conducted by Danyuo et al. 34 suggest that cancer drug release kinetics in breast cancer is non-Fickean.

A common characteristic of the occurrence of non-Fickean patterns, as suggested in several works [70, 84, 48, 67, 45], is the multi-scale and heterogeneous nature of the environment in which diffusion takes place. Specifically, Lacks 74 shows that geometric factors, such as tortuosity, could cause the diffusion processes occurring in a neuromuscular junction to be non-Fickean. Within this view, in the case of a tumour, although to our knowledge there is no experimental evidence that correlates non-Fickean diffusion with its internal structure, its microvascular network is known to have a strong influence on transport phenomena. In fact, this issue has been discussed in several papers, like e.g. 69, 90 and references therein.

In general, non-Fickean behaviours can be gathered in two categories:

(i) non-locality in time, which associates the mass flux of a given chemical agent with the concentration gradient of that agent through an integro-differential relationship, such as, for 
example, those involving fractional time derivatives or fractional time integrals [9];

(ii) non-locality in space, which means that the mass flux vector of a species cannot be expressed as a point-wise linear function of the concentration gradient, as Fick's law would prescribe.

In this work, we focus on the second type of non-locality, and we are interested in quantifying the spatial influence of the mass flux at a given point on "distant" points of a body. However, it is important to recall that non-locality is a broad notion [43, 47], which covers a wide spectrum of phenomena, from transport processes [44 to plasticity [2, 57] or visco-elasticity [10, 37], and depends on the intrinsic structure of the system to which it is referred and/or on its response to long-range stimuli. Moreover, non-locality can be introduced in different ways, e.g., by having recourse to higher-order gradient theories, as is the case for plasticity [2, 157, 109, or by assigning constitutive laws that feature integro-differential operators [72, 43. In particular, the employment of integrals and derivatives of fractional order [92, 9, 12] has demonstrated to be an effective method in the description of various non-local phenomena [11, 18, 22, including non-Fickean diffusion [26, 82, 35, [86. As pointed out in [35], the introduction of Fractional Calculus allows for the description of non-Fickean transport processes in a natural way, because of their close connection with the concept of anomalous diffusion [84].

Before going further, we notice that in the literature there exist other non-Fickean diffusion laws that, however, do not rely on the assumption of non-local effects. In particular, the MaxwellStefan model [71], which generalises Fick's diffusion by the consideration of "thermodynamic nonidealities" 1 and "influence of external force fields", has been postulated in the study of porous media and tumour growth 68.

\subsection{Aim and novelties of our work}

In the present work, on the basis of the indications given above, our aim is to highlight and study the influence of the non-local character of diffusion processes that could be acting in an avascular tumour. To accomplish this task, we propose a potentially new constitutive relationship of fractional type for the mass flux vector. Consequently, we refer only to fractional operators in space, so that the model is non-local in space but local in time. In our formulation, the mass flux vector of the chemical species, evaluated at a given spatial point, is put in relation, through an integral operator, to the concentration gradient of that species, evaluated at all other points of the region of space occupied by the tumour. This leads to a generalisation of Fick's law that can be related to Fractional Calculus in a straightforward manner. In particular, this connection will become evident in the specification of the mass flux vector for the study of a benchmark problem (see Section "Definition of the non-locality function").

For our purposes, we adopt part of the modelling assumptions outlined in [80, 101, 156, 91. Specifically, we study the tumour as a mixture comprising a fluid phase and a solid phase, and we identify its growth with the gain or loss of mass of the solid phase at the expenses or advantage of the fluid one. In particular, the model we employ predicts the gain of mass for a sufficiently high

\footnotetext{
${ }^{1}$ According to [115, the thermodynamic non-idealities are related to a phenomenon that pertains to a thermodynamic system, like, for instance, a gas, and that occurs through the "storage of potential energy" among the molecules of the system itself as a result of the interactions among such molecules. The main consequence of the non-idealities is that the concentrations of the molecules turn out to be different from those expected in the absence of the energy storage among them.
} 
concentration of chemical agents (in fact, nutrients) and the loss of mass when the concentration of these falls below a certain threshold [81, 80]. Moreover, in the case of mass uptake of the solid phase, the model accounts for mechanotransduction [81, 80, 50, 56, thereby allowing a modulation of growth by means of stress [81, 80], whereas both for positive and for negative growth, the onset of structural transformations and their related inelastic distortions are considered. In the remainder of this work, we address only the most pertinent considerations and equations, while we refer the Reader to [80, 101, 91 for further details.

Before going further, we find it convenient to highlight the main novelties of our work, which can be summarised as follows:

1. Impact of non-local diffusion on tumour growth. With respect to [80, 101, 56, 91], we study the diffusion of the chemical agents in a growing tumour by hypothesising a non-local constitutive law for the diffusive mass flux vector. This is done with the purpose of weighing how and to which extent the deviation of non-local diffusion from the Fickean one impacts on the main descriptors of the tumour's evolution.

2. Evolving non-locality driven by the tumour's dynamics. The model that we are proposing requires to solve a type of non-locality that changes with the dynamics of the tumour through its motion and growth. To the best of our knowledge, this is a generalisation of a setting adopted in several papers (see e.g. [35, 63, 105, 755), where the non-locality is accounted for in advection-diffusion equations without considering the deformation or structural change of the media in which such equations are defined.

3. Non-locality and non-linearity. The core of our work is the equation governing the evolution of chemical agents. This is given by an advection-diffusion-reaction equation featuring a fractional diffusive mass flux vector and a non-linear reaction term. We solve this equation together with all the other balance laws, expressed by non-linear partial differential equations, that model the tumour and its growth. Therefore, we solve a system of equations in which non-linearity combines with non-locality. To us, this is a novelty because, to the best of our knowledge, papers on Fractional Calculus usually solve one equation in conjunction with a fractional constitutive law. Furthermore, the nature of the problem we are tackling makes it impossible to have recourse to solution techniques based on Fourier and Laplace transforms, which are standard for problems of Fractional Calculus that are linear and/or formulated in unbounded domains. In our case, however, this assumption would be physically unrealistic and we have, thus, to turn to numerical techniques, such as Finite Element (FE) methods.

We point out that the study of fractional diffusion in bounded domains is delicate because of the complexity of the numerics involving operators of fractional type. Nevertheless, in the literature there exist some works dealing with fractional diffusion equations on bounded domains. The majority of these works employ finite-difference Grünwald-Letnikov discretisation schemes (see e.g. [88, 76, 36, 83]), and there also exist studies in which FE methods have been used for solving equations of fractional type [99, 63, 49, 44]. However, to the best of our knowledge, there is still a lack of studies addressing in detail the numerical issues arising in the context of fractional differential equations within a non-linear mechanical framework.

We also mention that, in this work, we suggest a possible way of formulating non-local diffusion on manifolds by adapting the definition of convolution on manifolds given in [106. Originally, we encountered the necessity of expressing convolution in the non-Euclidean context because we aimed 
at writing our model in fully covariant formalism as a first step towards non-Euclidean settings. However, we faced some technical difficulties, which made us opt, for the time being, to give just a sketch of the generalisation of non-local diffusion on manifolds. For this reason, we summarised the main steps of our generalisation in Appendix A1. Note that Meerschaert et al. 82 did consider diffusion-like problems on manifolds but within a different framework.

Finally, we would like to point out that, throughout this work, the terminologies "mass fraction" and "concentration" will be often used interchangeably, and the spatial and temporal dependence of the variables are dropped out, unless there is a necessity to account for the non-local character of the problem, where this dependence is explicitly specified.

\section{Kinematics}

Let $\mathscr{S}$ be the three-dimensional Euclidean space, $\mathscr{T}$ an interval of time, and $\mathscr{B} \subset \mathscr{S}$ the reference placement of the mechanical system representing an avascular tumour, in which the tumour may, or may not, be free of stress. In particular, we consider that the tumour is a saturated mixture comprising a solid and a fluid phase. Moreover, the region of $\mathscr{S}$ occupied by the system at time $t \in \mathscr{T}$ is referred to as current configuration and is denoted by $\mathscr{B}_{t} \equiv \chi(\mathscr{B}, t)$, where $\chi(\cdot, t): \mathscr{B} \rightarrow \mathscr{S}$ describes the motion of the solid phase (for the mixture kinematics, we follow here the same approach as the one adopted in 33]). Then, a point $x \in \mathscr{B}_{t}$ is given by $x=\chi(X, t)$, with $X \in \mathscr{B}$ and $t \in \mathscr{T}$. By differentiating the motion $\chi$ with respect to $X$, we obtain the deformation gradient tensor, $\boldsymbol{F}$, defined as the tangent map of $\chi$, i.e., $\boldsymbol{F}(\cdot, t) \equiv T \chi(\cdot, t): T \mathscr{B} \rightarrow T \mathscr{S}$, with $T \mathscr{B}=\sqcup_{X \in \mathscr{B}} T_{X} \mathscr{B}$ and $T \mathscr{S}=\sqcup_{x \in \mathscr{S}} T_{x} \mathscr{S}$. Thus, tensor $\boldsymbol{F}(X, t)$ characterises the visible deformations of the system by mapping vectors of the tangent space $T_{X} \mathscr{B}$ into the tangent space $T_{x} \mathscr{S}$.

We also introduce the spatial volumetric fractions of the solid and the fluid phases, given by $\varphi_{\mathrm{s}}(x, t)$ and $\varphi_{\mathrm{f}}(x, t)$, respectively. Then, we define the apparent mass densities, $\varphi_{\mathrm{s}}(x, t) \varrho_{\mathrm{s}}(x, t)$ and $\varphi_{\mathrm{f}}(x, t) \varrho_{\mathrm{f}}(x, t)$, of the solid and of the fluid, where $\varrho_{\mathrm{s}}(x, t)$ and $\varrho_{\mathrm{f}}(x, t)$ represent the true mass densities of the solid and the fluid phase, respectively. We notice that the apparent mass densities express, in each case, the phase mass per unit volume of the mixture as a whole, whereas each true mass density is the inherent density of the corresponding phase. Furthermore, the saturation of the mixture implies that $\varphi_{\mathrm{s}}(x, t)+\varphi_{\mathrm{f}}(x, t)=1$, for all $x \in \mathscr{B}_{t}$ and $t \in \mathscr{T}$.

The velocity of the mixture is $\boldsymbol{v}(x, t):=\sum_{\mathrm{k} \in\{\mathrm{s}, \mathrm{f}\}} \varphi_{\mathrm{k}}(x, t) \varrho_{\mathrm{k}}(x, t) \boldsymbol{v}_{\mathrm{k}}(x, t) / \varrho(x, t)$, where $\boldsymbol{v}_{\mathrm{s}}(x, t)$ and $\boldsymbol{v}_{\mathrm{f}}(x, t)$ denote the velocities of the solid and the fluid phases, respectively, and $\varrho(x, t):=$ $\sum_{\mathrm{k} \in\{\mathrm{s}, \mathrm{f}\}} \varphi_{\mathrm{k}}(x, t) \varrho_{\mathrm{k}}(x, t)$ is the mass density of the mixture as a whole. We notice that, by introducing the solid phase velocity $\boldsymbol{V}_{\mathrm{S}}(X, t):=\dot{\chi}(X, t)$, where the "dot" symbol denotes differentiation with respect to time, the relationship $\boldsymbol{v}_{\mathrm{s}}(x, t)=\boldsymbol{v}_{\mathrm{s}}(\chi(X, t), t)=\boldsymbol{V}_{\mathrm{S}}(X, t)$ holds true for all $X \in \mathscr{B}$ and $t \in \mathscr{T}$. Furthermore, since the tumour under study is assumed to be a mixture also in $\mathscr{B}$, the solid and the fluid coexist at every point $X \in \mathscr{B}$. This situation implies that any point $x$ in the fluid phase can be also viewed as the image of $X$ through the motion $\chi$ and, consequently, $\boldsymbol{v}_{\mathrm{f}}(x, t)=\boldsymbol{v}_{\mathrm{f}}(\chi(X, t), t)=\boldsymbol{V}_{\mathrm{f}}(X, t)$. 


\subsection{Kinematics of growth}

As suggested in several works, see e.g. [46, 110] and references therein, a relevant aspect in the growth of a tumour is the manifestation of irreversible changes of its internal structure. To take this aspect into account, we employ some concepts taken from the theory of inelastic processes. Specifically, for characterising the growth of the tissue under study, we invoke the Bilby-Kröner-Lee (BKL) decomposition of the deformation gradient tensor [85, 27, 102, 98, 52, i.e.,

$$
\boldsymbol{F}=\boldsymbol{F}_{\mathrm{e}} \boldsymbol{F}_{\gamma}
$$

where the generally non-integrable tensor fields $\boldsymbol{F}_{\mathrm{e}}$ and $\boldsymbol{F}_{\gamma}$ describe the elastic accommodation of the tumour and the inelastic distortions induced by growth, respectively. We denote by $\mathscr{N}_{t}(X)$ the natural state of the body element of the tumour's solid phase associated with $X$, and we let it represent a stress-free state. We refer to the tensor $\boldsymbol{F}_{\gamma}(X, t): T_{X} \mathscr{B} \rightarrow \mathscr{N}_{t}(X)$ as growth tensor and we assume that it comprehends the structural transformations undergone by the tumour in the course of its evolution. Then, the accommodating elastic tensor $\boldsymbol{F}_{\mathrm{e}}(X, t)$ maps vectors of $\mathscr{N}_{t}(X)$ into vectors of $T_{x} \mathscr{S}$. We refer to the works [102, [52, 94, 27, 101, [56], and references therein, for a more complete discussion on the nature and generalisation of the multiplicative decomposition in Equation (1).

In particular, following [80, 101, 56, in the present work we contemplate the case in which the growth tensor is a pure dilatation, that is, we impose $\boldsymbol{F}_{\gamma}=\gamma \boldsymbol{I}$, where $\gamma>0$ is referred to as growth parameter and $\boldsymbol{I}$ is the second-order identity tensor.

\section{Balance laws}

By adopting the modelling assumptions made in [80, 101, 56], we consider that the fluid phase is constituted by chemical agents and "water", with mass fractions $c_{\mathrm{a}}$ and $c_{\mathrm{w}}$, respectively, and such that $c_{\mathrm{a}}+c_{\mathrm{w}}=1$. Furthermore, we hypothesise the solid phase to consist of two type of cells, i.e., the proliferating cells, with mass fraction $c_{\mathrm{p}}$, and the necrotic cells, with mass fraction $c_{\mathrm{n}}$, where $c_{\mathrm{p}}+c_{\mathrm{n}}=1$.

\subsection{Mass balance laws}

The mass balance laws for the gain and loss of mass of the proliferating and the necrotic cells, and for the mass fraction of the chemical species and the fluid phase as a whole are

$$
\begin{aligned}
& \partial_{t}\left(\varphi_{\mathrm{s}} \varrho_{\mathrm{s}} c_{\mathrm{p}}\right)+\operatorname{div}\left(\varphi_{\mathrm{s}} \varrho_{\mathrm{s}} c_{\mathrm{p}} \boldsymbol{v}_{\mathrm{s}}\right)=r_{\mathrm{pn}}+r_{\mathrm{fp}}, \\
& \partial_{t}\left(\varphi_{\mathrm{s}} \varrho_{\mathrm{s}} c_{\mathrm{n}}\right)+\operatorname{div}\left(\varphi_{\mathrm{s}} \varrho_{\mathrm{s}} c_{\mathrm{n}} \boldsymbol{v}_{\mathrm{s}}\right)=r_{\mathrm{nf}}-r_{\mathrm{pn}}, \\
& \partial_{t}\left(\varphi_{\mathrm{f}} \varrho_{\mathrm{f}} c_{\mathrm{a}}\right)+\operatorname{div}\left(\varphi_{\mathrm{f}} \varrho_{\mathrm{f}} c_{\mathrm{a}} \boldsymbol{v}_{\mathrm{f}}+\boldsymbol{y}_{\alpha}\right)=r_{\mathrm{ap}}, \\
& \partial_{t}\left(\varphi_{\mathrm{f}} \varrho_{\mathrm{f}}\right)+\operatorname{div}\left(\varphi_{\mathrm{f}} \varrho_{\mathrm{f}} \boldsymbol{v}_{\mathrm{f}}\right)=-r_{\mathrm{s}},
\end{aligned}
$$

where $r_{\mathrm{pn}}, r_{\mathrm{fp}}, r_{\mathrm{nf}}$ and $r_{\mathrm{ap}}$ denote rates of mass intake and/or reduction [80, 101, 56]. Specifically, they represent the rate at which the proliferating cells turn into necrotic $\left(r_{\mathrm{pn}}\right)$, the mass from the fluid phase that promotes the proliferation of cells $\left(r_{\mathrm{fp}}\right)$, the necrotic cells that dissolve into the fluid $\left(r_{\mathrm{nf}}\right)$, and the chemical agents that are depleted by the proliferating cells $\left(r_{\mathrm{ap}}\right)$. Moreover, 
$r_{\mathrm{s}}:=r_{\mathrm{fp}}+r_{\mathrm{nf}}$ is the global source/sink of mass of the solid phase as a whole. Particularly, in writing Equations (2a) and (2b), we have enforced the consideration that the two cell populations move at the same velocity $\boldsymbol{v}_{\mathrm{s}}$. In Equation (2c), the term $\boldsymbol{y}_{\alpha}$ corresponds to the mass flux vector of the chemical agents, and since the focus of this work is subordinate to its definition, we prefer to make a deeper analysis of its characterisation and physical meaning in a separate section.

By enforcing that the tissue's cells are mainly composed by water [19, 180, 151, the true mass density of the solid phase, $\varrho_{\mathrm{S}}$, can be regarded as constant and equal to the true mass density of the fluid phase, $\varrho_{\mathrm{f}}$, which is set to be equal to the density of water. Thus, by taking into account the saturation constraint and the BKL decomposition in Equation (1), Equations (2a)-(2d), written with respect to the reference configuration, become

$$
\begin{aligned}
& \dot{\mathfrak{c}}_{\mathrm{p}}=\left[R_{\mathrm{pn}}+R_{\mathrm{fp}}-R_{\mathrm{s}} \mathfrak{c}_{\mathrm{p}}\right]\left[J_{\gamma} \Phi_{\mathrm{s} \nu} \varrho_{\mathrm{s}}\right]^{-1}, \\
& \frac{\dot{\gamma}}{\gamma}=\left[R_{\mathrm{fp}}+R_{\mathrm{nf}}\right]\left[3 \varrho_{\mathrm{s}} \Phi_{\mathrm{s} \nu} J_{\gamma}\right]^{-1}, \\
& \varrho_{\mathrm{f}}\left[J-J_{\gamma} \Phi_{\mathrm{s} \nu}\right] \dot{\mathfrak{c}}_{\mathrm{a}}+\varrho_{\mathrm{f}} \boldsymbol{Q} \operatorname{Grad} \mathfrak{c}_{\mathrm{a}}+\operatorname{Div} \boldsymbol{Y}_{\alpha}=\mathfrak{c}_{\mathrm{a}} R_{\mathrm{s}}+R_{\mathrm{ap}}, \\
& \operatorname{Div} \boldsymbol{Q}+\dot{J}=0,
\end{aligned}
$$

where the material filtration velocity $\boldsymbol{Q}$, the material mass flux vector of the chemical agents $\boldsymbol{Y}_{\alpha}$, the mass fractions $\mathfrak{c}_{\mathrm{a}}$ and $\mathfrak{c}_{\mathrm{p}}$, and the material sources/sinks of mass featuring in Equations (3a)-(3d) are given by

$$
\begin{aligned}
\boldsymbol{Q}(X, t) & :=J(X, t) \boldsymbol{q}(\chi(X, t), t) \boldsymbol{F}^{-\mathrm{T}}(X, t), \\
\boldsymbol{Y}_{\alpha}(X, t) & :=J(X, t) \boldsymbol{y}_{\alpha}(\chi(X, t), t) \boldsymbol{F}^{-\mathrm{T}}(X, t), \\
\mathfrak{c}_{\mathrm{k}}(X, t) & :=c_{\mathrm{k}}(\chi(X, t), t), \\
R_{\beta}(X, t) & :=J(X, t) r_{\beta}(\chi(X, t), t),
\end{aligned}
$$

$$
\begin{aligned}
& \mathrm{k} \in\{\mathrm{a}, \mathrm{p}\} \\
& \beta \in\{\mathrm{pn}, \mathrm{fp}, \mathrm{nf}, \mathrm{ap}, \mathrm{s}\},
\end{aligned}
$$

with $\boldsymbol{q}=\varphi_{\mathrm{f}}\left[\boldsymbol{v}_{\mathrm{f}}-\boldsymbol{v}_{\mathrm{s}}\right]$. We note that, in writing Equations (3a) $-(3 \mathrm{~d})$, the material volumetric fractions $\Phi_{\mathrm{s}}(X, t):=J(X, t) \varphi_{\mathrm{s}}(\chi(X, t), t)$ and $\Phi_{\mathrm{f}}(X, t):=J(X, t) \varphi_{\mathrm{f}}(\chi(X, t), t)$ have been written as $\Phi_{\mathrm{s}}=J_{\gamma} \Phi_{\mathrm{s} \nu}$ and $\Phi_{\mathrm{f}}=J-J_{\gamma} \Phi_{\mathrm{s} \nu}$, where $\Phi_{\mathrm{s} \nu}(X, t):=J_{\mathrm{e}}(X, t) \varphi_{\mathrm{s}}(\chi(X, t), t)$ is the "pull-back" of the solid phase volumetric fraction, $\varphi_{\mathrm{s}}$, to the natural state [101, 56]. In particular, by imposing that the temporal derivative of $J_{\gamma}$ compensates for the mass source $r_{\mathrm{s}}$ [42, [5], it can be deduced that the volumetric fraction $\Phi_{\mathrm{s} \nu}$ is independent of time. However, $\Phi_{\mathrm{s} \nu}$ may depend on material points [56]. Furthermore, since it holds true that $J_{\mathrm{e}}=J / J_{\gamma}$, the volumetric fractions of the solid and the fluid phase can be expressed entirely in terms of the volume ratios $J$ and $J_{\gamma}$, i.e.,

$$
\begin{aligned}
& \varphi_{\mathrm{s}}(x, t)=\varphi_{\mathrm{s}}(\chi(X, t), t)=\frac{J_{\gamma}(X, t) \Phi_{\mathrm{s} \nu}(X)}{J(X, t)}, \\
& \varphi_{\mathrm{f}}(x, t)=1-\varphi_{\mathrm{s}}(x, t)=\frac{J(X, t)-J_{\gamma}(X, t) \Phi_{\mathrm{s} \nu}(X)}{J(X, t)} .
\end{aligned}
$$

\subsection{Momentum balance laws}

In this work, we neglect inertial and body forces, so that the momentum balance laws for the biphasic medium as a whole and for the fluid phase write [60, 54, 91

$$
\operatorname{div}\left(\boldsymbol{\sigma}_{\mathrm{s}}+\boldsymbol{\sigma}_{\mathrm{f}}\right)=\mathbf{0},
$$




$$
\boldsymbol{q}=-\boldsymbol{k} \operatorname{grad} p,
$$

where $\boldsymbol{\sigma}_{\mathrm{s}}$ and $\boldsymbol{\sigma}_{\mathrm{f}}$ are the Cauchy stress tensors of the solid and the fluid phase, $p$ is the hydrostatic pressure, Equation (6b) expresses Darcy's law [60], and $\boldsymbol{k}$ denotes the permeability tensor, which is here taken to be symmetric and positive definite.

Following [60, 15, 53, 101, we assume the fluid phase to be macroscopically inviscid, so that $\boldsymbol{\sigma}_{\mathrm{f}}$ is purely hydrostatic, and we write

$$
\begin{aligned}
& \boldsymbol{\sigma}_{\mathrm{f}}=-\varphi_{\mathrm{f}} p \boldsymbol{g}^{-1} \\
& \boldsymbol{\sigma}_{\mathrm{s}}=-\varphi_{\mathrm{s}} p \boldsymbol{g}^{-1}+\boldsymbol{\sigma}_{\mathrm{sc}}
\end{aligned}
$$

where $\boldsymbol{\sigma}_{\mathrm{sc}}$ is said to be the constitutive part of $\boldsymbol{\sigma}_{\mathrm{s}}$ and $\boldsymbol{g}^{-1}$ is the inverse of the metric tensor, $\boldsymbol{g}$, associated with $\mathscr{S}$. Then, by substituting Equations (7a) and (7b) into Equation (6a), and performing the backward Piola transformation of Equations (6a) and (6b), we obtain

$$
\begin{aligned}
& \operatorname{Div}\left(-J \mathfrak{p} \mathfrak{g}^{-1} \boldsymbol{F}^{-\mathrm{T}}+\boldsymbol{P}_{\mathrm{sc}}\right)=\mathbf{0}, \\
& \boldsymbol{Q}=-\boldsymbol{K} \operatorname{Grad} \mathfrak{p},
\end{aligned}
$$

where we have introduced the notation

$$
\begin{aligned}
& \mathfrak{p}(X, t):=p(\chi(X, t), t), \\
& \boldsymbol{K}(X, t):=J(X, t) \boldsymbol{F}^{-1}(\chi(X, t), t) \boldsymbol{k}(\chi(X, t), t) \boldsymbol{F}^{-\mathrm{T}}(X, t), \\
& \boldsymbol{P}_{\mathrm{sc}}(X, t):=J(X, t) \boldsymbol{\sigma}_{\mathrm{sc}}(\chi(X, t), t) \boldsymbol{F}^{-\mathrm{T}}(X, t), \\
& \mathfrak{g}(X, t):=\boldsymbol{g}(\chi(X, t)),
\end{aligned}
$$

to denote, respectively, the pressure expressed as a function of time and of the points of $\mathscr{B}$, the material permeability tensor, the constitutive part of the overall first Piola-Kirchhoff stress tensor, and the metric tensor expressed as a function of time and of the points of $\mathscr{B}$. Moreover, Equation (8b) represents Darcy's law of filtration, pulled-back to the reference configuration.

\section{Constitutive laws I: Strain energy density and per- meability}

Following [80, 101, 56], we hypothesise that the solid phase of the tumour is isotropic and hyperelastic, and introduce the strain energy densities $\mathcal{W}$ and $\mathcal{W}_{\nu}$, which are written per unit volume of the reference configuration and of the natural state, respectively. To account for the structural changes induced by growth, the strain energy density $\mathcal{W}$ is expressed as a constitutive function, namely $\check{\mathcal{W}}$, depending on $\boldsymbol{F}, \boldsymbol{F}_{\gamma}$ and on material points. Furthermore, we denote by $\check{\mathcal{W}}_{\nu}$ the constitutive representation of $\mathcal{W}_{\nu}$, which is supposed here to depend solely on the tensor $\boldsymbol{F}_{\mathrm{e}}$. Therefore, the following relationship holds [42, 30, 101 .

$$
\check{\mathcal{W}}\left(\boldsymbol{F}(X, t), \boldsymbol{F}_{\gamma}(X, t), X\right)=J_{\gamma}(X, t) \check{\mathcal{W}}_{\nu}\left(\boldsymbol{F}_{\mathrm{e}}(X, t)\right) .
$$

Within a more general framework, the strain energy density $\check{\mathcal{W}}_{\nu}$ maintains the explicit dependence on $X$, and Equation (10) does not hold in its present form. This becomes evident when $\check{\mathcal{W}}_{\nu}$ is 
parameterised by point-dependent material coefficients or, by expressing $\check{\mathcal{W}}_{\nu}$ as $\check{\mathcal{W}}_{\nu}=\Phi_{\mathrm{s} \nu} \varrho_{\mathrm{s}} \check{\Psi}_{\mathrm{s}}$, where $\check{\Psi}_{\mathrm{s}}$ is the solid phase strain energy density per unit mass, when $\Phi_{\mathrm{s} \nu}$ depends on $X$. However, these circumstances are excluded from the setting of this work, as can be deduced by looking at Table 1, in which all the material parameters and $\Phi_{\mathrm{s} \nu}$ are taken as constants.

Hereafter, we adopt a constitutive law of the type proposed in 62 for $\check{\mathcal{W}}_{\nu}$, i.e.,

$$
\begin{aligned}
& \check{\mathcal{W}}_{\nu}\left(\boldsymbol{F}_{\mathrm{e}}\right)=\hat{\mathcal{W}}_{\nu}\left(\boldsymbol{C}_{\mathrm{e}}\right)=a_{0}\left\{\exp \left(\hat{\Psi}\left(\boldsymbol{C}_{\mathrm{e}}\right)\right)-1\right\}, \\
& \hat{\Psi}\left(\boldsymbol{C}_{\mathrm{e}}\right)=a_{1}\left[\hat{I}_{1}\left(\boldsymbol{C}_{\mathrm{e}}\right)-3\right]+a_{2}\left[\hat{I}_{2}\left(\boldsymbol{C}_{\mathrm{e}}\right)-3\right]-a_{3} \log \left(\hat{I}_{3}\left(\boldsymbol{C}_{\mathrm{e}}\right)\right),
\end{aligned}
$$

where $\hat{\mathcal{W}}_{\nu}$ is the constitutive representation of $\mathcal{W}$ expressed as a function of the elastic, right CauchyGreen deformation tensor $\boldsymbol{C}_{\mathrm{e}}=\boldsymbol{F}_{\mathrm{e}}^{\mathrm{T}} \cdot \boldsymbol{F}_{\mathrm{e}}=\boldsymbol{F}_{\gamma}^{-\mathrm{T}} \boldsymbol{C} \boldsymbol{F}_{\gamma}^{-1}, \boldsymbol{C}=\boldsymbol{F}^{\mathrm{T}} \cdot \boldsymbol{F}$ is the "classical", right CauchyGreen deformation tensor, $\hat{I}_{1}\left(\boldsymbol{C}_{\mathrm{e}}\right)=\operatorname{tr}\left(\boldsymbol{C}_{\mathrm{e}}\right), \hat{I}_{2}\left(\boldsymbol{C}_{\mathrm{e}}\right)=\frac{1}{2}\left\{\left[\hat{I}_{1}\left(\boldsymbol{C}_{\mathrm{e}}\right)\right]^{2}-\operatorname{tr}\left[\left(\boldsymbol{C}_{\mathrm{e}}\right)^{2}\right]\right\}$, and $\hat{I}_{3}\left(\boldsymbol{C}_{\mathrm{e}}\right)=$ $\operatorname{det}\left(\boldsymbol{C}_{\mathrm{e}}\right)$ are the principal invariants of $\boldsymbol{C}_{\mathrm{e}}$, and, as in [62, 114, 101], the parameters $a_{0}, a_{1}, a_{2}$ and $a_{3}$ are expressed in terms of Lamé's parameters $\lambda$ and $\mu$, i.e.,

$$
a_{0}=\frac{2 \mu+\lambda}{4 a_{3}}, \quad a_{1}=a_{3} \frac{2 \mu-\lambda}{2 \mu+\lambda}, \quad a_{2}=a_{3} \frac{\lambda}{2 \mu+\lambda}, \quad a_{3}=a_{1}+2 a_{2}=1 .
$$

Then, by using Equations (11a) and (11b), the constitutive part of the first Piola-Kirchhoff stress tensor reads 101

$$
\boldsymbol{P}_{\mathrm{sc}}=J_{\gamma} \boldsymbol{F} \boldsymbol{F}_{\gamma}^{-1}\left(2 \frac{\partial \hat{\mathcal{W}}_{\nu}}{\partial \boldsymbol{C}_{\mathrm{e}}}\left(\boldsymbol{C}_{\mathrm{e}}\right)\right) \boldsymbol{F}_{\gamma}^{-\mathrm{T}}
$$

Furthermore, we require the permeability tensor to be "unconditionally isotropic" [13], i.e., $\boldsymbol{k}=k_{0} \boldsymbol{g}^{-1}$, so that the material permeability tensor reads

$$
\boldsymbol{K}=J k_{0} \boldsymbol{C}^{-1} .
$$

In Equation (14), $k_{0}$ denotes the scalar permeability and is taken here as in [13, 62], i.e.,

$$
k_{0}=k_{\mathrm{R}}\left[\frac{J-J_{\gamma} \Phi_{\mathrm{s} \nu}}{J_{\gamma} \varphi_{\mathrm{fR}}}\right]^{m_{0}} \exp \left(\frac{m_{1}}{2}\left[\frac{J^{2}-J_{\gamma}^{2}}{J_{\gamma}^{2}}\right]\right),
$$

where $m_{0}$ and $m_{1}$ are constant material coefficients, $\varphi_{\mathrm{fR}}:=1-\Phi_{\mathrm{S} \nu}$ is a reference value of the fluid phase volumetric fraction, and $k_{\mathrm{R}}$ is the reference permeability of the medium. In the sequel, both $k_{\mathrm{R}}$ and $\varphi_{\mathrm{fR}}$, and thus $\Phi_{\mathrm{s} \nu}$, are assumed to be constant.

\section{Constitutive Laws II: Non-Fickean diffusion}

As pointed out in the Introduction, our aim is to generalise previous models of tumour growth [80, 101 by using some of the notions and tools offered by the theory of Fractional Calculus [92, 9, 12. To this end, we introduce a non-Fickean type of diffusion of the chemical agents. Specifically, our purpose is to take into account the non-local behaviour of the gradient of the chemical agents' mass fraction, and study its influence on the growth of an avascular tumour. 


\subsection{Non-Fickean mass flux vector}

We propose to express the chemical species' mass flux vector, $\boldsymbol{y}_{\alpha}$ (see Equation (2c)), in terms of a non-local constitutive law of convolution type, in which, in the Euclidean case, the kernel of the convolution integral features a power law in the distance between the points $x$ and $\tilde{x}$ of each pair $(x, \tilde{x})$ of spatial points occupied by body points. This way, we aim to show how $\boldsymbol{y}_{\alpha}$, evaluated at $x$, depends on the gradients of concentration evaluated at all other points $\tilde{x}$, and on the power law chosen for the convolution kernel. To do this, we face two difficulties: the first one is connected to the fact that, since, for the sake of generality, we view the body as a manifold, the concept of convolution has to be suitably generalised; the second one is due to the impossibility of integrating vector fields on manifolds. Whereas the first issue has been investigated in the literature [17, 106, 93, and we refer to the convolution on manifolds put forward in [106], the second issue can be circumvented by re-defining the mass flux vector of the chemical agents in weak form, i.e., for each $t \in \mathscr{T}$, we define $\boldsymbol{y}_{\alpha}$ through the duality product [16]

$$
\begin{aligned}
\left\langle\boldsymbol{y}_{\alpha}, \operatorname{grad} \check{c}\right\rangle & :=-\varrho_{\mathrm{f}} \int_{\mathscr{B}_{t}}\left\{\int_{\mathscr{B}_{t}}[\operatorname{grad} \check{c}(x)] \boldsymbol{d}_{\alpha}(x, \tilde{x}, t)\left[\operatorname{grad} c_{\mathrm{a}}(\tilde{x}, t)\right] \operatorname{dv}(\tilde{x})\right\} \operatorname{dv}(x), \\
\boldsymbol{d}_{\alpha}(x, \tilde{x}, t) & :=\mathfrak{f}_{\alpha}(x, \tilde{x}) \boldsymbol{d}_{\alpha}(x, \tilde{x}, t),
\end{aligned}
$$

for all $\check{c} \in \check{\mathcal{C}}=\left\{\check{c} \in H^{1}\left(\mathscr{B}_{t}\right): \check{c}=0\right.$ on $\left.\left(\partial \mathscr{B}_{t}\right)_{\mathrm{D}}\right\}$, with $\check{\mathcal{C}}$ being the space of all virtual variations of the mass fractions, $\left(\partial \mathscr{B}_{t}\right)_{\mathrm{D}}$ the portion of the boundary of $\mathscr{B}_{t}$ on which Dirichlet conditions are applied for the mass fraction of the chemical agents, and $H^{1}\left(\mathscr{B}_{t}\right)$ is the standard Sobolev space of square-integrable functions over $\mathscr{B}_{t}$ whose weak derivatives up to the order one are square-integrable over $\mathscr{B}_{t}$ too.

We refer to the second-order tensor $\boldsymbol{d}_{\alpha}(x, \tilde{x}, t)$ as non-local diffusivity tensor, and we express it as the product of the scalar quantity $\mathfrak{f}_{\alpha}(x, \tilde{x})$ and of the tensor $\mathfrak{d}_{\alpha}(x, \tilde{x}, t)$. In particular, for a given $x \in \mathscr{B}_{t}$ and varying $\tilde{x} \in \mathscr{B}_{t}, \mathfrak{f}_{\alpha}(x, \tilde{x})$, referred to as the non-locality function, measures how the intensity of the chemical signal expressed by $\operatorname{grad} c_{\mathrm{a}}(\tilde{x}, t)$ is felt at $x$. The tensor $\mathfrak{d}_{\alpha}(x, \tilde{x}, t)$, instead, is denominated fractional diffusivity tensor. We emphasise that $\mathfrak{f}_{\alpha}$ is defined for $x \neq \tilde{x}$ and that, since we are dealing with fractional diffusion, both $\mathfrak{d}_{\alpha}(x, \tilde{x}, t)$ and $\boldsymbol{d}_{\alpha}(x, \tilde{x}, t)$ have, in general, physical dimensions different from those of the standard diffusivity tensor, depending on the prescription of $\mathfrak{f}_{\alpha}$ and $\alpha \in \mathbb{R}^{+}$.

The way in which $\mathfrak{f}_{\alpha}(x, \tilde{x})$ is to be understood in the case in which $\mathscr{B}_{t}$ is viewed as a manifold is reported in Appendix A1. However, from here on, to avoid the technical difficulties of addressing such a general framework, which is out of the scope of this work, we prefer to adopt orthogonal Cartesian coordinates. Then, by regarding $\mathscr{B}_{t}$ as a flat subset of $\mathscr{S}$ having the same dimensionality as $\mathscr{S}, \mathfrak{f}_{\alpha}(x, \tilde{x})$ can be recast in the form $\mathfrak{f}_{\alpha}(x, \tilde{x})=\hat{\mathfrak{f}}_{\alpha}(x-\tilde{x})$, where $\hat{\mathfrak{f}}_{\alpha}$ is introduced to re-define $\mathfrak{f}_{\alpha}$ as a function of the vector $x-\tilde{x}$, i.e., as $\hat{\mathfrak{f}}_{\alpha}: T_{\tilde{x}} \mathscr{S} \rightarrow \mathbb{R}$ (see Appendix A1). Furthermore, we require $\mathfrak{d}_{\alpha}(x, \tilde{x}, t)$ to be a two-point tensor of the type $\mathfrak{d}_{\alpha}(x, \tilde{x}, t)=\sum_{a, b=1}^{3}\left[\mathfrak{d}_{\alpha}(x, \tilde{x}, t)\right]^{a b} \boldsymbol{e}_{a}(x) \otimes \boldsymbol{e}_{b}(\tilde{x})$, where $\left\{\boldsymbol{e}_{l}(x)\right\}_{l=1}^{3}$ and $\left\{\boldsymbol{e}_{l}(\tilde{x})\right\}_{l=1}^{3}$ are the vector bases attached to $x$ and $\tilde{x}$. It is worth noticing that, within a Cartesian setting, and for $x=\tilde{x}$, the tensor $\boldsymbol{e}_{a}(x) \otimes \boldsymbol{e}_{b}(\tilde{x}) \equiv \boldsymbol{e}_{a}(x) \otimes \boldsymbol{e}_{b}(x)$ is referred to as "Jacoby directional tensor" in 3, where, in a slightly different context, the central Marchaud fractional derivative is extended to the case of two- or three-dimensional problems.

In general, there is no correlation at all between the vector bases $\left\{\boldsymbol{e}_{l}(x)\right\}_{l=1}^{3}$ and $\left\{\boldsymbol{e}_{l}(\tilde{x})\right\}_{l=1}^{3}$ and, in fact, each basis can be chosen arbitrarily and independently of the other one. Nevertheless, $\left\{\boldsymbol{e}_{l}(\tilde{x})\right\}_{l=1}^{3}$ can be enforced to be the result of the parallel transport of $\left\{\boldsymbol{e}_{l}(x)\right\}_{l=1}^{3}$ along the geodesic 
connecting $x$ and $\tilde{x}$. In particular, in the Euclidean case, the arch of the geodesic connecting $x$ and $\tilde{x}$ is the segment of the straight line directed from $x$ to $\tilde{x}$ and the parallel transport of $\left\{\boldsymbol{e}_{l}(x)\right\}_{l=1}^{3}$ along such a line renders $\left\{\boldsymbol{e}_{l}(\tilde{x})\right\}_{l=1}^{3}$ collinear with $\left\{\boldsymbol{e}_{l}(x)\right\}_{l=1}^{3}$. Hence, for each $l=1,2,3, \boldsymbol{e}_{l}(x)$ and $\boldsymbol{e}_{l}(\tilde{x})$ can be associated with the same direction, hereafter denoted by $\boldsymbol{i}_{l}$, even though they remain, implicitly, distinct vectors, attached to different spatial points. Within this approach, we hypothesise that $\mathfrak{d}_{\alpha}(x, \tilde{x}, t)$ admits the representation $\mathfrak{d}_{\alpha}(x, \tilde{x}, t)=\sum_{b=1}^{3} \mathfrak{d}_{\alpha}^{b}(x, \tilde{x}, t) \boldsymbol{e}_{b}(x) \otimes \boldsymbol{e}_{b}(\tilde{x})$ and, since $\boldsymbol{e}_{l}(x)$ is collinear with $\boldsymbol{e}_{l}(\tilde{x})$, this representation of $\mathfrak{d}_{\alpha}(x, \tilde{x}, t)$ mimics the description of an orthotropic tensor function with respect to the set of directions $\left\{\boldsymbol{i}_{1}, \boldsymbol{i}_{2}, \boldsymbol{i}_{3}\right\}$. Hence, it is "as if" we had $\mathfrak{d}_{\alpha}(x, \tilde{x}, t)=\sum_{b=1}^{3} \mathfrak{d}_{\alpha}^{b}(x, \tilde{x}, t) \boldsymbol{i}_{b} \otimes \boldsymbol{i}_{b}$. Then, by using the definitions in Equation (16), we identify the components of the fractional mass flux to be given by the following expression

$$
\left[\boldsymbol{y}_{\alpha}(x, t)\right]^{b}:=-\varrho_{\mathrm{f}} \int_{\mathscr{B}_{t}} \hat{\mathfrak{f}}_{\alpha}(x-\tilde{x}) \mathfrak{d}_{\alpha}^{b}(x, \tilde{x}, t) \partial_{b} c_{\mathrm{a}}(\tilde{x}, t) \operatorname{dv}(\tilde{x}), \quad \text { no sum over } b=1,2,3,
$$

and we call the coefficients $\left\{\mathfrak{d}_{\alpha}^{b}(x, \tilde{x}, t)\right\}_{b=1}^{3}$ fractional diffusivities.

\subsection{Comparison with other works}

Other definitions of fractional mass flux vector can be found that characterise non-Fickean diffusion processes (see e.g. [82, 105] and references therein). For instance, Sapora et al. [105] study a fractional version of Darcy's law in one dimension in which the filtration velocity (also known as "specific mass flux") is taken to be proportional to an integral operator that the Authors refer to as "Riesz integral" [105] of pressure (note that the definition of Riesz integral given in [105 differs by a factor $\cos (\beta \pi / 2)$, with $\beta \in] 0,1$ [, from that in [104, 9]). However, when passing to higher dimensionalities, it is necessary to extend the concept of fractional differentiation to other differential operators like the gradient of a scalar function. In this regard, in [40, 1, 113] the fractional gradient of order $\alpha \in \mathbb{R}^{+}$of a scalar function is defined as a co-vector, whose components are identified with the fractional partial derivatives, each of which of order $\alpha$, of the given function. In particular, these fractional partial derivatives are taken in the sense of Riemann-Liouville in [40] and in the sense of Caputo in [113], whereas the Nishimoto fractional derivative [87] is used in [1], for $\alpha \in] 0,1]$.

For the purposes of our work, we adopt the definition given in Equation (17). This definition presents some fundamental differences with respect to the definition supplied, for instance, in [105]. These differences, however, are not only related to the fact that the physical phenomenon addressed in 105 is distinct from the one we are studying here. Rather, they are intrinsic in the definition of the operator expressing $\boldsymbol{y}_{\alpha}$, and can be summarised as follows:

- Equation (17) is conceived in a three-dimensional setting and, consequently, requires an integration over the whole configuration of the body, $\mathscr{B}_{t}$, whereas the definition of the mass flux given in [105] features an integration over a bounded interval.

- In our definition, each fractional diffusivity $\mathfrak{d}_{\alpha}^{b}(x, \tilde{x}, t), b=1,2,3$, is part of the integrand of Equation (17), and cannot be factorised out of the corresponding integral.

- If, for a given $b_{0} \in\{1,2,3\}$, the fractional diffusivity $\mathfrak{d}_{\alpha}^{b_{0}}(x, \tilde{x}, t)$ could be factorised out of the integral in Equation (17) (e.g. by setting $\mathfrak{d}_{\alpha}^{b_{0}}(x, \tilde{x}, t) \equiv \mathfrak{d}_{0 \alpha}$, with $\mathfrak{d}_{0 \alpha}$ constant), and if 
the only nonzero component of $\operatorname{grad} c(\tilde{x}, t)$ were $\partial_{b_{0}} c_{\mathrm{a}}(\tilde{x}, t)$ for all $\tilde{x}$ and $t$, one would have

$$
\left[\boldsymbol{y}_{\alpha}(x, t)\right]^{b_{0}}=-\varrho_{\mathrm{f}} \mathfrak{d}_{0 \alpha} \int_{\mathscr{B}_{t}} \hat{\mathfrak{f}}_{\alpha}(x-\tilde{x}) \partial_{b_{0}} c_{\mathrm{a}}(\tilde{x}, t) \mathrm{dv}(\tilde{x}),
$$

where $\hat{\mathfrak{f}}_{\alpha}(x-\tilde{x})$ is still a function of all the components of the vector $x-\tilde{x}$, rather than of its $b_{0}$-th component only. This property marks a major difference between our approach and the model developed in [105], and expresses the fact that, even in the presence of a preferred direction (i.e., the one associated with $\partial_{b_{0}} c_{\mathrm{a}}$ ), one should account for the non-locality in all directions.

Before going further, we notice that, if the fractional diffusivities $\left\{\mathfrak{d}_{\alpha}^{b}(x, \tilde{x}, t)\right\}_{b=1}^{3}$ are all equal to some reference constant value $\mathfrak{d}_{\mathrm{R} \alpha}$ (note that, for simplicity, we call 'fractional diffusivities' the set of the three principal fractional diffusivities), the mass flux vector $\boldsymbol{y}_{\alpha}(x, t)$ can be expressed (in a Cartesian setting) as

$$
\boldsymbol{y}_{\alpha}(x, t)=-\varrho_{\mathrm{f}} \mathfrak{d}_{\mathrm{R} \alpha} \int_{\mathscr{B} t} \hat{\mathfrak{f}}_{\alpha}(x-\tilde{x}) \operatorname{grad} c_{\mathrm{a}}(\tilde{x}, t) \mathrm{dv}(\tilde{x}) .
$$

Moreover, for some suitable $\hat{\mathfrak{f}}_{\alpha}(x-\tilde{x})$, usually written as a power-law that decays in space, the integral on the right-hand-side of Equation (19) can be taken as the definition of a fractional gradient of $c_{\mathrm{a}}$ of order $\alpha$, i.e., one can write (in the Cartesian setting)

$$
\begin{aligned}
\operatorname{grad}^{\alpha} c_{\mathrm{a}}(x, t) & :=\int_{\mathscr{B}_{t}} \hat{\mathfrak{f}}_{\alpha}(x-\tilde{x}) \operatorname{grad} c_{\mathrm{a}}(\tilde{x}, t) \operatorname{dv}(\tilde{x}), \\
\left.\operatorname{grad}^{\alpha} c_{\mathrm{a}}(x, t)\right]_{b} & :=\int_{\mathscr{B}_{t}} \hat{\mathfrak{f}}_{\alpha}(x-\tilde{x}) \partial_{b} c_{\mathrm{a}}(\tilde{x}, t) \operatorname{dv}(\tilde{x}), \quad b=1,2,3 .
\end{aligned}
$$

Equations (20a) and (20b) are reminiscent of the definition of fractional gradient of order $\alpha$ supplied in [113. However, an important difference between that definition and ours is that, in [113], the components of the fractional gradient of $c_{\mathrm{a}}$ (i.e., $\left\{\left[\operatorname{grad}^{\alpha} c_{\mathrm{a}}(x, t)\right]_{b}\right\}_{b=1}^{3}$ in our notation) are identified with the Caputo derivatives of $c_{\mathrm{a}}$ along the principal directions of the vector basis. This, in turn, requires the function $\hat{\mathfrak{f}}_{\alpha}$ of Tarasov [113] to depend, for each Caputo derivative, solely on the $b$-th component of $x-\tilde{x}$.

\subsection{Backward Piola transform of the mass flux vector}

The backward Piola transformation of Equation 1 (16a) is given by

$$
\begin{aligned}
\left\langle\boldsymbol{y}_{\alpha}, \operatorname{grad} \check{c}\right\rangle & =\left\langle\boldsymbol{Y}_{\alpha}, \operatorname{Grad} \check{\mathfrak{c}}\right\rangle \\
& =-\varrho_{\mathrm{f}} \int_{\mathscr{B}}\left\{\int_{\mathscr{B}}[\operatorname{Grad} \check{\mathfrak{c}}(X, t)] \boldsymbol{D}_{\alpha}(X, \tilde{X}, t)\left[\operatorname{Grad} \mathfrak{c}_{\mathrm{a}}(\tilde{X}, t)\right] \mathrm{d} V(\tilde{X})\right\} \mathrm{dV}(X),
\end{aligned}
$$

with $\check{\mathfrak{c}}$ and $\mathfrak{c}_{\mathrm{a}}$ such that $\check{\mathfrak{c}}(X, t)=\check{c}(\chi(X, t))$ and $\mathfrak{c}_{\mathrm{a}}(X, t)=c_{\mathrm{a}}(\chi(X, t), t)$, and we introduced the material non-local diffusivity tensor, $\boldsymbol{D}_{\alpha}$, the material non-locality function, $\mathfrak{F}_{\alpha}$, and the material fractional diffusivity tensor, $\mathfrak{D}_{\alpha}$, as follows

$$
\boldsymbol{D}_{\alpha}(X, \tilde{X}, t):=J(X, t) \mathfrak{F}_{\alpha}(X, \tilde{X}, t) \mathfrak{D}_{\alpha}(X, \tilde{X}, t),
$$




$$
\begin{aligned}
\mathfrak{F}_{\alpha}(X, \tilde{X}, t) & :=\hat{\mathfrak{f}}_{\alpha}(\chi(X, t)-\chi(\tilde{X}, t)), \\
\mathfrak{D}_{\alpha}(X, \tilde{X}, t) & :=J(\tilde{X}, t) \boldsymbol{F}^{-1}(\chi(X, t), t) \mathfrak{d}_{\alpha}(\chi(X, t), \chi(\tilde{X}, t), t) \boldsymbol{F}^{-\mathrm{T}}(\tilde{X}, t) .
\end{aligned}
$$

390

More specifically, the components of $\mathfrak{D}_{\alpha}(X, \tilde{X}, t)$ and $\boldsymbol{Y}_{\alpha}(X, t)$ are given by

$$
\begin{aligned}
{\left[\mathfrak{D}_{\alpha}(X, \tilde{X}, t)\right]^{A B} } & =J(\tilde{X}, t) \sum_{b=1}^{3}\left[\boldsymbol{F}^{-1}(\chi(X, t), t)\right]_{b}^{A} \mathfrak{d}_{\alpha}^{b}(\chi(X, t), \chi(\tilde{X}, t), t)\left[\boldsymbol{F}^{-\mathrm{T}}(\tilde{X}, t)\right]_{b}{ }^{B}, \\
{\left[\boldsymbol{Y}_{\alpha}(X, t)\right]^{A} } & =-\varrho_{\mathrm{f}} \int_{\mathscr{B}} J(X, t) \mathfrak{F}_{\alpha}(X, \tilde{X}, t) \sum_{B=1}^{3}\left[\mathfrak{D}_{\alpha}(X, \tilde{X}, t)\right]^{A B} \partial_{B} \mathfrak{c}_{\mathrm{a}}(\tilde{X}, t) \mathrm{dV}(\tilde{X}) .
\end{aligned}
$$

Expression (23b) defines the components of the mass flux vector in the material description, whereas $\mathfrak{D}_{\alpha}$ is the material counterpart of the fractional diffusivity tensor $\mathfrak{d}_{\alpha}$.

In the sequel, we assume the spatial fractional diffusivities to be all equal to each other, i.e., $\mathfrak{d}_{\alpha}^{b}(x, \tilde{x}, t)=\mathfrak{d}_{\alpha}(x, \tilde{x}, t)$, for all $b=1,2,3$, and that $\mathfrak{d}_{\alpha}(x, \tilde{x}, t)$ is independent of $x$ (more rigorously, we should say that $\mathfrak{d}_{\alpha}$ can be redefined as a function of time and of the spatial variable with respect to which the integration is made, i.e., $\tilde{x}$ ). Consequently, with a slight abuse of notation, we simply write $\mathfrak{d}_{\alpha}(\tilde{x}, t)$. Moreover, following [101], we impose that $\mathfrak{d}_{\alpha}(\tilde{x}, t)$ depends on position and time through the volumetric fraction of the fluid phase, thereby setting $\mathfrak{d}_{\alpha}(\tilde{x}, t)=\varphi_{\mathrm{f}}(\tilde{x}, t) \mathfrak{d}_{\mathrm{R} \alpha}$, where $\mathfrak{d}_{\mathrm{R} \alpha}$ is a reference fractional diffusivity, which is parameterised by $\alpha$. Since $\varphi_{\mathrm{f}}(\tilde{x}, t)$ can be related to the volumetric deformation of the solid phase and to growth through the expression (5b), we obtain

$$
\mathfrak{d}_{\alpha}(\chi(\tilde{X}, t), t)=\frac{J(\tilde{X}, t)-J_{\gamma}(\tilde{X}, t) \Phi_{\mathrm{s} \nu}}{J(\tilde{X}, t)} \mathfrak{d}_{\mathrm{R} \alpha} .
$$

These considerations imply that the components of $\mathfrak{D}_{\alpha}$ can be written as follows

$$
\left[\mathfrak{D}_{\alpha}(X, \tilde{X}, t)\right]^{A B}=\left(J(\tilde{X}, t)-J_{\gamma}(\tilde{X}, t) \Phi_{\mathrm{s} \nu}\right) \mathfrak{d}_{\mathrm{R} \alpha}\left[\boldsymbol{F}^{-1}(\chi(X, t), t)\right]_{b}^{A}\left[\boldsymbol{F}^{-\mathrm{T}}(\tilde{X}, t)\right]_{b}{ }^{B} .
$$

We notice that the non-local nature of the problem is also reflected in Equation (25). Indeed, in a model accounting only for local interactions, the last two terms of Equation (25) would give the inverse of the right Cauchy-Green deformation tensor $\boldsymbol{C}$, i.e., $\boldsymbol{C}^{-1}=\boldsymbol{F}^{-1} \cdot \boldsymbol{F}^{-\mathrm{T}}$, since $X$ and $\tilde{X}$ would coincide. Still, this is not true in our case, since the non-locality changes with the dynamics of the tissue. Moreover, even in the case in which all the fractional diffusivities $\left\{\mathfrak{d}_{\alpha}^{b}(x, \tilde{x}, t)\right\}_{b=1}^{3}$ were independent of $x$ and $\tilde{x}$, their material counterparts $\left\{\left[\mathfrak{D}_{\alpha}(X, \tilde{X}, t)\right]^{A B}\right\}_{A, B=1}^{3}$ would still be functions of the points $X$ and $\tilde{X}$ because of the motion, $\chi$.

Remark 1 Due to the non-local nature of the mass flux vector, its Piola transformation needs to be performed in two steps, i.e., as many as the integrals appearing in Equation (16a), or Equation (21). In particular, the volume ratio $J(X, t)$ is due to the change of measure of the outermost integral of Equation (21), which re-defines the duality product between $\boldsymbol{y}_{\alpha}$ and gradč into the duality product between $\boldsymbol{Y}_{\alpha}$ and Graď́. In our formalism, this volume ratio is used to define the pull-back of the non-local diffusivity tensor, $\boldsymbol{d}_{\alpha}$, as prescribed by Equations (22a)-(22c). Furthermore, the tensor $\boldsymbol{F}^{-1}(\chi(X, t), t)$ featuring in Equation (22c) stems from the transformation of the gradient of the virtual concentration, $\check{c}$, evaluated at $x$, i.e., $\operatorname{grad} \check{c}(\chi(X, t), t)=\operatorname{Grad} \check{c}(X, t) \boldsymbol{F}^{-1}(\chi(X, t), t)$, 
and it contributes, "from the left", to the calculation of the pull-back of the fractional diffusivity tensor. Whereas this first part of the backward Piola transformation of the mass flux vector is standard, the second part of it reveals the non-locality of the constitutive law in Equation (21). Indeed, the tensor $\boldsymbol{F}^{-\mathrm{T}}(\tilde{X}, t)$ featuring in Equation (22c) must be evaluated in $\tilde{X}$ because it originates from the transformation of the gradient of the concentration (not the virtual one), which is part of the integrand of the innermost integral, i.e., the one expressing the non-local constitutive law. This tensor contributes, "from the right", to determine the pull-back of the fractional diffusivity tensor. Finally, the volume ratio $J(\tilde{X}, t)$ is necessary because of the change of measure in the innermost integral of Equation (16a) and is employed to define the pull-back of the fractional diffusivity tensor, $\mathfrak{d}_{\alpha}$. In conclusion, to determine the pull-back of the mass flux vector, a "double" Piola transformation has to be performed.

Remark 2 Looking at the Piola transformation of the mass flux vector, it is worth mentioning that the non-locality of the problem, expressed through $\hat{\mathfrak{f}}_{\alpha}$ as a function of $(x-\tilde{x})$ in the current configuration, cannot be described in general as a function of $(X-\tilde{X})$ in the reference configuration. Rather, the material non-locality function, $\mathfrak{F}_{\alpha}$, must be conceived as a function of the three variables $X, \tilde{X}$ and $t$ since, as prescribed by Equation (22b), it inherits this dependence from the motion, $\chi$, in a way that, in general, cannot be reduced to a function of time and of the difference $(X-\tilde{X})$. Furthermore, we notice that the non-locality of the problem evolves from the reference to the current configuration. Indeed, two points that are "close" in $\mathscr{B}$ can either be "far away" from each other or become "even closer" in $\mathscr{B}_{t}$, and vice versa.

\section{Model summary and some numerical aspects}

In this section, we summarise the equations characterising our mathematical model, specify the expressions for the sinks and sources of mass, and highlight some computational aspects to be taken into account. In the following, we focus on the case in which the considered chemical agents are nutrient substances that are necessary to trigger and maintain the growth of the tumour. Hence, we shall be referring to "nutrients" in lieu of "chemical agents" from here on.

\subsection{Model equations}

Our model is based on the following set of non-linear and coupled equations

$$
\begin{aligned}
& \dot{\mathfrak{c}}_{\mathrm{p}}=\left[R_{\mathrm{pn}}+R_{\mathrm{fp}}-R_{\mathrm{s}} \mathfrak{c}_{\mathrm{p}}\right]\left[J_{\gamma} \Phi_{\mathrm{s} \nu} \varrho_{\mathrm{s}}\right]^{-1}, \\
& \frac{\dot{\gamma}}{\gamma}=\left[R_{\mathrm{fp}}+R_{\mathrm{nf}}\right]\left[3 \varrho_{\mathrm{s}} \Phi_{\mathrm{s} \nu} J_{\gamma}\right]^{-1}, \\
& \varrho_{\mathrm{f}}\left[J-J_{\gamma} \Phi_{\mathrm{s} \nu}\right] \dot{\mathfrak{c}}_{\mathrm{a}}-\varrho_{\mathrm{f}}[\boldsymbol{K} \operatorname{Grad} \mathfrak{p}] \operatorname{Grad} \mathfrak{c}_{\mathrm{a}}+\operatorname{Div} \boldsymbol{Y}_{\alpha}=\mathfrak{c}_{\mathrm{a}} R_{\mathrm{s}}+R_{\mathrm{ap}}, \\
& \dot{J}-\operatorname{Div}(\boldsymbol{K} \operatorname{Grad} \mathfrak{p})=0, \\
& \operatorname{Div}\left(-J \mathfrak{p} \mathfrak{g}^{-1} \boldsymbol{F}^{-\mathrm{T}}+\boldsymbol{P}_{\mathrm{sc}}\right)=\mathbf{0},
\end{aligned}
$$


in the $(4+3)$ unknowns $\mathscr{U}:=\left\{\mathfrak{c}_{\mathrm{p}}, \gamma, \mathfrak{c}_{\mathrm{a}}, \mathfrak{p},\left\{\chi^{a}\right\}_{a=1}^{3}\right\}$, and with the source and sink terms [80, 101, 81]

$$
\begin{aligned}
& R_{\mathrm{fp}}=J \zeta_{\mathrm{fp}}\left\langle\frac{\mathfrak{c}_{\mathrm{a}}-\mathfrak{c}_{\mathrm{cr}}}{\mathfrak{c}_{\mathrm{env}}-\mathfrak{c}_{\mathrm{cr}}}\right\rangle_{+}\left[1-\frac{\delta_{1}\langle\bar{\sigma}\rangle_{+}}{\delta_{2}+\langle\bar{\sigma}\rangle_{+}}\right] \underbrace{\frac{J-J_{\gamma} \Phi_{\mathrm{s} \nu}}{J \varphi_{\mathrm{fR}}}}_{=\varphi_{\mathrm{f}} / \varphi_{\mathrm{fR}}} \underbrace{J_{\gamma} \Phi_{\mathrm{s} \nu}}_{=\varphi_{\mathrm{s}}} \mathfrak{c}_{\mathrm{p}}, \\
& R_{\mathrm{nf}}=-J \zeta_{\mathrm{nf}} \frac{J_{\gamma} \Phi_{\mathrm{s} \nu}}{J}\left(1-\mathfrak{c}_{\mathrm{p}}\right), \\
& R_{\mathrm{ap}}=-J \zeta_{\mathrm{ap}} \frac{\mathfrak{c}_{\mathrm{a}}}{\mathfrak{c}_{\mathrm{a}}+\mathfrak{c}_{0}} \frac{J_{\gamma} \Phi_{\mathrm{s} \nu}}{J} \mathfrak{c}_{\mathrm{p}}, \\
& R_{\mathrm{pn}}=-J \zeta_{\mathrm{pn}}\left\langle 1-\frac{\mathfrak{c}_{\mathrm{a}}}{\mathfrak{c}_{\mathrm{cr}}}\right\rangle_{+} \frac{J_{\gamma} \Phi_{\mathrm{s} \nu}}{J} \mathfrak{c}_{\mathrm{p}} .
\end{aligned}
$$

In Equations 27a $-27 \mathrm{c}), \zeta_{\mathrm{fp}}, \zeta_{\mathrm{nf}}, \zeta_{\mathrm{ap}}$ and $\zeta_{\mathrm{pn}}$ are constants indicating the characteristic time scales with which the interstitial fluid is absorbed by the proliferating cells, the necrotic cells go into the fluid, nutrients are consumed, and proliferating cells die, respectively. The operator $\langle f\rangle_{+}:=\max \{0, f\}$ represents Macaulay's brackets, which return the positive part of a function $f$. Moreover, $\mathfrak{c}_{\mathrm{cr}}$ is a critical value for the nutrients' mass fraction and $\mathfrak{c}_{\text {env }}$ refers to the concentration of nutrients present in the surrounding of the tumour. In order for growth to occur, it is necessary that $R_{\mathrm{fp}}=J r_{\mathrm{fp}}>0$, i.e., it must hold that $\mathfrak{c}_{\mathrm{a}}>\mathfrak{c}_{\mathrm{cr}}$, provided $\mathfrak{c}_{\mathrm{env}}>\mathfrak{c}_{\mathrm{cr}}$. We also mention that the mass source $R_{\mathrm{fp}}$ features the term in square brackets depending on $\bar{\sigma}:=-\frac{1}{3} \operatorname{tr} \sigma$, which is introduced in order to describe the fact that growth can be modulated by mechanical stress, thereby giving rise to a phenomenon known as mechanotransduction [81, 80, 50, 56]. Finally, the product of the last three factors in Equation (27a) describes the fact that, to allow for the transfer of mass from the fluid to the proliferating cells, there must be a nonzero volumetric fraction of the fluid phase and of the solid phase as well as a nonzero mass fraction of the proliferating cells. Macaulay's brackets in Equation (27d) ensure that the proliferating cells become necrotic, i.e., $R_{\mathrm{pn}}<0$ when $\mathfrak{c}_{\mathrm{a}}<\mathfrak{c}_{\mathrm{cr}}$, and $R_{\mathrm{pn}}=0$ otherwise. Equation (27b) assumes that $R_{\mathrm{nf}}$ is linear in the volumetric fraction of the solid phase and in the mass fraction of the necrotic cells, i.e., $1-\mathfrak{c}_{\mathrm{p}}$, while $R_{\text {ap }}$ establishes that the magnitude with which the nutrients are "eaten" by the proliferating cells depends on the ratio $\mathfrak{c}_{a} / \mathfrak{c}_{0}$, with $\left.\left.\mathfrak{c}_{0} \in\right] 0,1\right]$ being a reference value of the nutrients' concentration that modulates their consumption. We refer the Reader to [81, 80, 101, 56] for further details on these terms, and for their generalisation to include growth-induced structural transformations.

Finally, we recall that the main goal of our model is to quantify the impact of the non-local diffusion of the nutrients, accounted for by $\boldsymbol{Y}_{\alpha}$, on the overall evolution of the tumour, i.e., on all the unknowns of the model. We note that, apart from the presence of the fractional mass flux vector $\boldsymbol{Y}_{\alpha}$, our model is the same as the one presented in [80] and extended in [101, 56].

\subsection{Numerical aspects}

The model summarised in Equation (26) features ordinary differential equations, partial differential equations and an integro-differential equation of fractional type. Since the model is formulated for a bounded domain and many couplings and nonlinearities are accounted for, the usual techniques adopted in Fractional Calculus for linear problems, such as the Fourier and Laplace transforms, cannot be used. Consequently, we need to resort to numerical techniques. In particular, we solve 
Equations (26a) - (26e) by means of a FE scheme that we need to adapt to our purposes in order to take fractional derivatives into account. Here, we do not intend to go into the details of the numerical scheme, which is out of the scope of this work. Nevertheless, we intend to give some insights about the most important computational aspects of our work, while the numerical solutions are obtained by using COMSOL Multiphysics(R).

Classical FE techniques [55, 103 have been used for solving numerically Equations (26a), 26b), (26d) and (26e), while Equation (26c) has required a special care. To this end, we report explicitly only the weak formulation corresponding to it. Before doing this, we denote with $(\partial \mathscr{B})_{\mathrm{D}}$ and $(\partial \mathscr{B})_{\mathrm{N}}$ the Dirichlet and Neumann boundaries of $\mathscr{B}$, respectively, and assume $\partial \mathscr{B}=(\partial \mathscr{B})_{\mathrm{D}} \sqcup(\partial \mathscr{B})_{\mathrm{N}}$. Furthermore, by using the standard formalism for Sobolev spaces [16, and using the space of virtual concentrations, $\check{\mathcal{C}}_{\mathrm{R}}:=\left\{\check{\mathfrak{c}} \in H^{1}(\mathscr{B})\right.$ s.t. $\left.\left.\check{\mathfrak{c}}\right|_{(\partial \mathscr{B})_{\mathrm{D}}}=0\right\}$, we have that, for all $\check{\mathfrak{c}} \in \check{\mathcal{C}}_{\mathrm{R}}$, the following weak form applies

$$
\begin{gathered}
\int_{\mathscr{B}}\left\{\varrho_{\mathrm{f}}\left[J-J_{\gamma} \Phi_{\mathrm{s} \nu}\right] \dot{\mathfrak{c}}_{\mathrm{a}}-\varrho_{\mathrm{f}}[\boldsymbol{K} \operatorname{Grad} \mathfrak{p}] \operatorname{Grad} \mathfrak{c}_{\mathrm{a}}-\mathfrak{c}_{\mathrm{a}} R_{\mathrm{s}}-R_{\mathrm{ap}}\right\} \check{\mathfrak{c}} \mathrm{dV} \\
-\int_{\mathscr{B}} \boldsymbol{Y}_{\alpha} \mathrm{Grad} \check{\mathfrak{c}} \mathrm{dV}+\int_{(\partial \mathscr{B})_{\mathrm{N}}} \boldsymbol{Y}_{\alpha} \cdot \boldsymbol{N} \check{\mathfrak{c}} \mathrm{dS}=0,
\end{gathered}
$$

where $\boldsymbol{N}$ is the field of unit vectors normal to $(\partial \mathscr{B})_{\mathrm{N}}$ while $\boldsymbol{Y}_{\alpha}$ is given in Equation (21), so that the second volume integral of Equation (28) (without the sign) becomes

$$
\begin{aligned}
& \int_{\mathscr{B}} \boldsymbol{Y}_{\alpha}(X, t) \operatorname{Grad} \check{\mathfrak{c}}(X, t) \mathrm{dV}(X) \\
& =-\varrho_{\mathrm{f}} \int_{\mathscr{B}}\left\{\int_{\mathscr{B}}[\operatorname{Grad} \check{\mathfrak{c}}(X, t)] \boldsymbol{D}_{\alpha}(X, \tilde{X}, t)\left[\operatorname{Gradc} \mathfrak{c}_{\mathrm{a}}(\tilde{X}, t)\right] \mathrm{dV}(\tilde{X})\right\} \mathrm{dV}(X) .
\end{aligned}
$$

After applying a backward Euler scheme for the time derivative, a linearisation procedure, and Galerkin method, Equation (28) leads to a system of algebraic equations that, except for a non-local stiffness matrix, arising from the double integral in Equation (29), is similar to the one obtained in standard FE approaches. From a numerical point of view, the non-local stiffness matrix reflects a long range coupling among the elements in the spatial discretisation. Indeed, it is worth noting that, in the construction of the non-local stiffness matrix, the cross integrations between the piecewise polynomial ansatz functions do not vanish as they would in the case of the stiffness matrix of a standard diffusion problem. That is, even though two discretisation nodes are far away from each other, the entry of the matrix corresponding to these nodes will be non-zero, because of the presence of the non-locality function $\hat{\mathfrak{f}}_{\alpha}$. This results into stiffness matrices that are denser, the stronger the non-locality is. In fact, this is a typical feature of the numerical study of non-local differential equations based on the use of FE methods (see for instance [47]). Still, as pointed out in [47], standard techniques for the solution of such equations, like Gauss elimination, can be used.

Before closing this section, we would like to remark that, in the simulations carried out in our work, the stiffness matrix associated with Equation $(29)$ is symmetric and positive definite. 


\section{Benchmark problem and considerations on the non- locality function}

In this section, we specify a benchmark problem in order to simplify and solve the mathematical model given by Equations (26a)-(26e). To this end, we make use of the problem proposed in [5], and recently investigated in [101, 56] to account for growth-induced inelastic distortions. By doing this, we intend to model the volumetric growth of an avascular tumour in a "jacketed" cylindrical sample (its deformation is restricted to be along the longitudinal axis only), and to investigate, how and to what extent, the non-local diffusivity properties of the nutrients influence the dynamics of the tissue. In the following, we assume that the problem complies with axial symmetry and that it is radially homogeneous regardless of how slender the cylindrical sample is. This will require suitable a priori restrictions on all the unknowns of the problem.

\subsection{Description of the benchmark problem}

As in [101, 56], we adopt the cylindrical coordinates $(R, \Theta, Z)$ and $(r, \vartheta, z)$, associated with the reference and the current configurations of the tumour, respectively. Moreover, we require the motion to satisfy with the conditions

$$
\begin{aligned}
& \chi^{r}(R, \Theta, Z, t)=r=R, \\
& \chi^{\vartheta}(R, \Theta, Z, t)=\vartheta=\Theta, \\
& \chi^{z}(R, \Theta, Z, t)=z=Z+u(Z, t),
\end{aligned}
$$

where $u$ is the unknown axial component of displacement. In this situation, the tumour is allowed to expand itself solely along the axial direction and $\chi^{z}$ is the only unknown component of the motion, $\chi$. Additionally, to comply with the axial symmetry and with the radial homogeneity of the problem, the pressure $\mathfrak{p}$ is considered to be a function of the axial coordinate and time only. Another restriction pertains to the growth parameter $\gamma$, which is also assumed to depend only on $Z$ and $t$ (note that since the growth tensor $\boldsymbol{F}_{\gamma}=\gamma \boldsymbol{I}$ is spherical, it maintains the symmetries of the problem). Similar requirements also apply for the mass fraction of the proliferating cells, $\mathfrak{c}_{\mathrm{p}}$, as well as for the mass fraction of the nutrients, $\mathfrak{c}_{\mathrm{a}}$.

The motion we have assumed implies that the matrix representations of the deformation gradient tensor $\boldsymbol{F}$ and of the right Cauchy-Green deformation tensor $\boldsymbol{C}$ read

$$
\begin{aligned}
& {[\boldsymbol{F}]=\operatorname{diag}\left\{1,1,1+u^{\prime}\right\},} \\
& {[\boldsymbol{C}]=\operatorname{diag}\left\{1,1,\left[1+u^{\prime}\right]^{2}\right\},}
\end{aligned}
$$

where $u^{\prime}$ denotes the derivative of $u$ in the axial direction. Since it holds that $J=\operatorname{det}(\boldsymbol{F})=1+u^{\prime}>$ $0, u^{\prime}$ must obey the inequality $u^{\prime}>-1$.

Additionally, the growth tensor admits the diagonal form

$$
\left[\boldsymbol{F}_{\gamma}\right]=\operatorname{diag}\{\gamma, \gamma, \gamma\}, \quad \gamma>0,
$$

and, consequently, the elastic right Cauchy-Green deformation tensor $\boldsymbol{C}_{\mathrm{e}}$ has the representation

$$
\left[\boldsymbol{C}_{\mathrm{e}}\right]=\operatorname{diag}\left\{\frac{1}{\gamma^{2}}, \frac{1}{\gamma^{2}}, \frac{\left[1+u^{\prime}\right]^{2}}{\gamma^{2}}\right\} \text {. }
$$


Because of Equations (31a), (31b), (32) and (33), of the symmetry properties of the pressure term $-J \mathfrak{p g}^{-1} \boldsymbol{F}^{-\mathrm{T}}$, and of the constitutive expression (13), the first Piola-Kirchhoff stress tensor $\boldsymbol{P}=-J \mathfrak{p g}^{-1} \boldsymbol{F}^{-\mathrm{T}}+\boldsymbol{P}_{\mathrm{sc}}$ has the diagonal representation

$$
[\boldsymbol{P}]=\operatorname{diag}\left\{-J \mathfrak{p}+\left[\boldsymbol{P}_{\mathrm{sc}}\right]^{r R},-J \mathfrak{p}+\left[\boldsymbol{P}_{\mathrm{sc}}\right]^{\vartheta \Theta},-\mathfrak{p}+\left[\boldsymbol{P}_{\mathrm{sc}}\right]^{z Z}\right\},
$$

where each quantity featuring in each component of $\boldsymbol{P}$ is a function solely of $Z$ and time. Moreover, it applies that $\left[\boldsymbol{P}_{\mathrm{sc}}\right]^{r R}=\left[\boldsymbol{P}_{\mathrm{sc}}\right]^{\vartheta \Theta}$ and, thus, the balance of linear momentum (26e) in cylindrical coordinates reduces to

$$
\frac{\partial}{\partial Z}\left(-\mathfrak{p}+\left[\boldsymbol{P}_{\mathrm{sc}}\right]^{Z Z}\right)=0 .
$$

This result can be found also in other benchmark problems, such as the confined compression tests of articular cartilage, under symmetry assumptions similar to those made here. Therefore, Equation (35) constitutes a simplification obtained by virtue of symmetry and not by invoking the slenderness of the cylinder used in our benchmark (see Table 1).

Note also that, according to Equations (14) and (15), the conditions imposed on the deformation and on the growth tensor are such that $k_{0}$ depends, through $J$ and $J_{\gamma}$, only on the axial coordinate and on time. Moreover, the same conclusion can be drawn for the diffusivity $\mathfrak{d}_{\alpha}$, which, with slight abuse of notation, we express as $\mathfrak{d}_{\alpha}(Z, t)$ from here on.

By following the same reasoning that has led to Equation (35), and noticing that the only non-zero component of the mass flux $\boldsymbol{Q}$ is the axial one, i.e., $\vec{Q}^{Z}=-\boldsymbol{K}^{Z Z} \frac{\partial p}{\partial Z}$ with $\boldsymbol{K}^{Z Z}=$ $J k_{0}\left[\boldsymbol{C}^{-1}\right]^{Z Z}=k_{0} /\left(1+u^{\prime}\right)$, the continuity equation (26d) becomes

$$
\frac{\partial^{2} u}{\partial Z \partial t}-\frac{\partial}{\partial Z}\left(\frac{k_{0}}{1+u^{\prime}} \frac{\partial \mathfrak{p}}{\partial Z}\right)=0 .
$$

The equations for $\mathfrak{c}_{\mathrm{p}}$ and $\gamma$, that is Equations (26a) and (26b), are scalar ODEs, and the fact that $\mathfrak{c}_{\mathrm{p}}$ and $\gamma$ depend only of $Z$ and $t$ is consistent with the symmetry properties of all the terms featuring in these equations. That said, a remark is in order for Equation (26b) to emphasise that the considered benchmark problem remains three-dimensional in spite of the axial symmetry and radial homogeneity that it enjoys. Indeed, looking at the source $R_{\mathrm{fp}}$ in Equation (27a), we notice that the mechanotransduction term (i.e., the term between brackets in Equation (27a)) features the trace of Cauchy stress tensor, which requires the evaluation of all the stress components, i.e., also of those in the radial and circumferential directions, these being non null because the cylinder is laterally jacketed. Therefore, we conclude that, even though the cylinder used for our benchmark problem is slender, with slenderness ratio $2 \cdot 10^{-2}$ (see the geometric data in Table 11), the problem itself necessitates to account for all the geometrical dimensions.

The last equation to consider is the balance law for $\mathfrak{c}_{\mathrm{a}}$ (see Equation (26c)) in which the non-standard mass flux $\boldsymbol{Y}_{\alpha}$ features, at least in principle, all the coordinates (i.e., also the radial and the circumferential coordinates) through the non-locality function $\mathfrak{F}_{\alpha}(X, \tilde{X}, t)=\hat{\mathfrak{f}}_{\alpha}(\chi(X, t)-$ $\chi(\tilde{X}, t))$. To maintain the axial symmetry of the problem and to eliminate the dependence of the nutrients' mass flux on the radial and circumferential coordinates, two paths may be followed. One is discussed in Section "Definition of the non-locality function" and, for consistency with the symmetry requirements introduced so far, it imposes to rephrase the non-locality function as a function of the axial coordinate only. However, another path - valid for the problem at hand- 
could be to eliminate the dependence of the non-locality function on the radial and circumferential coordinate by taking advantage of the slenderness of the cylinder. To this end, we write the nonlocality function as

$$
\hat{\mathfrak{f}}_{\alpha}(x-\tilde{x})=\mathfrak{f}_{0 \alpha} \frac{1}{\|x-\tilde{x}\|^{\alpha}}=\mathfrak{f}_{0 \alpha} \frac{1}{\left\|(z-\tilde{z}) \boldsymbol{e}_{z}+\boldsymbol{r}_{t}\right\|^{\alpha}},
$$

where $\boldsymbol{e}_{z}$ is the unit vector along which the cylinder's axis is directed, $\mathfrak{f}_{0 \alpha}$, with $\left.\alpha \in\right] 0,1[$, is an $\alpha$-dependent coefficient to be individuated, and $\boldsymbol{r}_{t}$ is a vector lying on the cross-section of the cylinder. Next, we rescale the axial vector $(z-\tilde{z}) \boldsymbol{e}_{z}$ by the undeformed length of the cylinder, i.e., $2 L_{\text {in }}$, and the transverse vector $\boldsymbol{r}_{t}$ by the cylinder diameter prior to deformation, i.e., $2 R_{\text {in }}$, so that Equation (37) becomes

$$
\hat{\mathfrak{f}}_{\alpha}(x-\tilde{x})=\mathfrak{f}_{0 \alpha} \frac{1}{\left\|2 L_{\text {in }} \boldsymbol{\rho}_{a}+2 R_{\text {in }} \boldsymbol{\rho}_{t}\right\|^{\alpha}}=\frac{\mathfrak{f}_{0 \alpha}}{\left(2 L_{\text {in }}\right)^{\alpha}} \frac{1}{\left\|\boldsymbol{\rho}_{a}+\left(R_{\text {in }} / L_{\text {in }}\right) \boldsymbol{\rho}_{t}\right\|^{\alpha}},
$$

with $\boldsymbol{\rho}_{a}=(z-\tilde{z}) \boldsymbol{e}_{z} /\left(2 L_{\text {in }}\right)$ and $\boldsymbol{\rho}_{t}:=\boldsymbol{r}_{t} /\left(2 R_{\text {in }}\right)$. Now, since the slenderness ratio $R_{\text {in }} / L_{\text {in }}$ is $2 \cdot 10^{-2}$, we assume, within the first approximation, that the non-locality function can be truncated at the zero-th order in the slenderness ratio, thereby taking the expression

$$
\hat{\mathfrak{f}}_{\alpha}(x-\tilde{x}) \approx \frac{\mathfrak{f}_{0 \alpha}}{\left(2 L_{\mathrm{in}}\right)^{\alpha}} \frac{1}{\left\|\boldsymbol{\rho}_{a}\right\|^{\alpha}}=\mathfrak{f}_{0 \alpha} \frac{1}{\left\|(z-\tilde{z}) \boldsymbol{e}_{z}\right\|^{\alpha}}=\mathfrak{f}_{0 \alpha} \frac{1}{|z-\tilde{z}|^{\alpha}} .
$$

As discussed below, the coefficient $\mathfrak{f}_{0 \alpha}$ acquires the meaning of a normalisation factor.

\subsection{Initial and boundary conditions}

To solve Equations (26a)-(26e), we impose the same boundary and initial conditions used in [101, 56]. Specifically, at the initial instant of time we consider a reference configuration being characterised by the following relations

$$
\chi^{r}(R, \Theta, Z, 0)=R, \quad \chi^{\vartheta}(R, \Theta, Z, 0)=\Theta, \quad \chi^{z}(R, \Theta, Z, 0)=Z,
$$

where $R \in\left[0, R_{\mathrm{in}}\left[, \Theta \in\left[0,2 \pi\left[\right.\right.\right.\right.$ and $Z \in\left[-L_{\mathrm{in}},+L_{\mathrm{in}}\right]$, while $R_{\mathrm{in}}$ and $2 L_{\mathrm{in}}$ denote the radius and the length of the undeformed specimen. Besides, we enforce that, at $t=0$, necrotic cells are absent, i.e., $\mathfrak{c}_{\mathrm{p}}(R, \Theta, Z, 0)=1$, the fluid pressure is zero, i.e., $\mathfrak{p}(R, \Theta, Z, 0)=0$, the nutrients' mass fraction equals the environmental one, i.e., $\mathfrak{c}_{\mathrm{a}}(R, \Theta, Z, 0)=\mathfrak{c}_{\mathrm{env}}>0$, and the distribution of the growth parameter is homogeneous and unitary, i.e., $\gamma(R, \Theta, Z, 0)=1$. In addition, we consider the following boundary conditions

$$
\begin{aligned}
& \left(-J \mathfrak{p} \mathfrak{g}^{-1} \boldsymbol{F}^{-\mathrm{T}}+\boldsymbol{P}_{\mathrm{sc}}\right) \cdot \boldsymbol{N}_{\mathrm{A}}=\mathbf{0}, \\
& (-\boldsymbol{K} \mathrm{Grad} \mathfrak{p}) \cdot \boldsymbol{N}_{\mathrm{C}}=0, \\
& \mathfrak{p}=0, \\
& \mathfrak{c}_{\mathrm{a}}=\mathfrak{c}_{\mathrm{env}}, \\
& \boldsymbol{Y}_{\alpha} \cdot \boldsymbol{N}_{\mathrm{C}}=0,
\end{aligned}
$$

$$
\begin{aligned}
& \text { on }(\partial \mathscr{B})_{\text {Left }} \text { and }(\partial \mathscr{B})_{\text {Right }} \text {, } \\
& \text { on }(\partial \mathscr{B})_{\mathrm{C}} \text {, } \\
& \text { on }(\partial \mathscr{B})_{\text {Left }} \text { and }(\partial \mathscr{B})_{\text {Right }} \text {, } \\
& \text { on }(\partial \mathscr{B})_{\text {Left }} \text { and }(\partial \mathscr{B})_{\text {Right }} \text {, } \\
& \text { on }(\partial \mathscr{B})_{\mathrm{C}} \text {, }
\end{aligned}
$$

where $\boldsymbol{N}_{\mathrm{A}}$ and $\boldsymbol{N}_{\mathrm{C}}$ are fields of unit vectors normal to $(\partial \mathscr{B})_{\text {Left }} \cup(\partial \mathscr{B})_{\text {Right }}$ and to $(\partial \mathscr{B})_{\mathrm{C}}$, respectively, and $\partial \mathscr{B}=(\partial \mathscr{B})_{\text {Left }} \cup(\partial \mathscr{B})_{\text {Right }} \cup(\partial \mathscr{B})_{\mathrm{C}}$. Specifically, $(\partial \mathscr{B})_{\text {Left }}$ and $(\partial \mathscr{B})_{\text {Right }}$ are the left and the right surfaces at the extremities of $\mathscr{B}$, and $(\partial \mathscr{B})_{\mathrm{C}}$ is the lateral boundary. 


\subsection{Definition of the non-locality function}

A classical approach for defining $\hat{\mathfrak{f}}_{\alpha}$ is to adopt a power-law that decays in space. To our knowledge, this is customary for problems that are a priori formulated as one-dimensional and in which $\hat{\mathfrak{f}}_{\alpha}(x-\tilde{x})$ is assumed to be proportional to the reciprocal of $|x-\tilde{x}|^{\alpha}$, with $x$ and $\tilde{x}$ being points of the real line or of an interval of finite length [112, 11, 108, 22, 105]. This choice permits to "import", with slight modifications, the definitions of the fractional derivatives in time (see e.g. 9]) to the fractional differentiation in space. However, in some situations it is necessary to assess an a priori relationship between the dimensionality of the problem under study and the non-locality that must - or may - be resolved, once the dimensionality has been settled. Indeed, in a three-dimensional problem endowed with the symmetry and homogeneity properties we are dealing with, the only non-zero partial derivative of the concentration is the one along the axial direction. In such a situation, the axial mass flux reads

$$
\left[\boldsymbol{y}_{\alpha}(x, t)\right]^{z}=-\varrho_{\mathrm{f}} \int_{\mathscr{B}_{t}} \hat{\mathfrak{f}}_{\alpha}(x-\tilde{x}) \mathfrak{d}_{\alpha}(\tilde{z}, t) \partial_{\tilde{z}} c_{\mathrm{a}}(\tilde{z}, t) \mathrm{dv}(\tilde{x}),
$$

whereas the radial and the circumferential components of the flux are zero. Note that we are using here the customary formalism for cylindrical coordinates, so that $\tilde{x}=(\tilde{r}, \tilde{\vartheta}, \tilde{z})$. As anticipated before, the expression for $\left[\boldsymbol{y}_{\alpha}(x, t)\right]^{z}$ reminds the definition of fractional gradient given in [113, with the difference that a volume integral is used in (42) and that all the components of $x-\tilde{x}$ are considered.

In spite of the fact that the problem is one-dimensional from the point of view of its symmetries, the axial flux is still determined by an integration over the three-dimensional region $\mathscr{B}_{t}$, and $\hat{\mathfrak{f}}_{\alpha}(x-\tilde{x})$ describes, as it stands, a non-locality in three dimensions (trivially, because $x-\tilde{x}$ is a vector of a three-dimensional vector space). Therefore, the component of $(x-\tilde{x})$ along the radial or the circumferential direction will influence the axial mass flux, even though the problem was claimed to enjoy axial symmetry and to be independent of the radial coordinate. This result, however, may be physically unsound. Indeed, one would expect non-locality to be coherent with the symmetries of the problem, even though the integral of Equation (42) is over the whole configuration $\mathscr{B}_{t}$, thereby maintaining the physical dimensionality of the problem itself.

To address this issue, we need to take into account how the symmetries of the problem under investigation influence the non-locality in the relationship between $\boldsymbol{y}_{\alpha}$ and $c_{\mathrm{a}}$. Consequently, the non-locality function $\hat{\mathfrak{f}}_{\alpha}$ in Equation (42) is re-defined as

$$
\left.\hat{\mathfrak{f}}_{\alpha}(x-\tilde{x}):=\hat{\mathfrak{h}}_{\alpha}(z-\tilde{z})=\frac{1}{\mathcal{N}(\alpha)} \frac{1}{|z-\tilde{z}|^{\alpha}}, \quad \alpha \in\right] 0,1[,
$$

where $\mathcal{N}(\alpha)$ is a normalisation factor to be determined. From Equations (42) and (43), we notice that the physical dimensions of the fractional diffusivity, $\mathfrak{d}_{\alpha}$, are $L^{1+\alpha} T^{-1}$, where $L$ and $T$ stand for the characteristic "length" and the characteristic "time" of the non-local diffusion process, respectively. Thus, when $\alpha$ tends to 1 (from below), we recover the physical dimensions of the standard diffusivity.

By substituting Equation (43) into Equation (42), and recalling that $\left.\mathscr{B}_{t}=\mathscr{C}_{\mathrm{R}} \times\right]-\ell(t),+\ell(t)[$ (where $\mathscr{C}_{\mathrm{R}}$ is the cross-section of the cylinder and $2 \ell(t)$ is its variable axial length), we obtain the much simpler expression

$$
\left[\boldsymbol{y}_{\alpha}(x, t)\right]^{z} \equiv y_{\alpha}^{z}(z, t)=-\frac{\varrho_{\mathrm{f}} \pi R_{\mathrm{in}}^{2}}{\mathcal{N}(\alpha)} \int_{-\ell(t)}^{+\ell(t)} \frac{1}{|z-\tilde{z}|^{\alpha}} \mathfrak{d}_{\alpha}(\tilde{z}, t) \partial_{\tilde{z}} c_{\mathrm{a}}(\tilde{z}, t) \mathrm{d} \tilde{z} .
$$


For the Equation (44) to be physically sound, it has to return the axial component of the standard mass flux vector in the limit $\alpha \rightarrow 1^{-}$. Unfortunately, proving this result for problems defined over bounded domains is not possible without knowing $c_{\mathrm{a}}$. On the contrary, this difficulty does not arise in problems defined over unbounded domains, because, with the aid of the Fourier transform, it is possible to do the following reasoning:

- Introduce the auxiliary notation $\psi_{\alpha}^{z}(\tilde{z}, t):=-\varrho_{\mathrm{f}} \mathfrak{d}_{\alpha}(\tilde{z}, t) \partial_{z} c_{\mathrm{a}}(\tilde{z}, t)$, and assume to prolong $y_{\alpha}^{z}(z, t)$ to the whole real line, so that Equation (44) becomes

$$
\begin{aligned}
y_{\alpha}^{z}(z, t) & =-\frac{\varrho_{\mathrm{f}} \pi R_{\text {in }}^{2}}{\mathcal{N}(\alpha)} \int_{-\infty}^{+\infty} \frac{1}{|z-\tilde{z}|^{\alpha}} \mathfrak{d}_{\alpha}(\tilde{z}, t) \partial_{\tilde{z}} c_{\mathrm{a}}(\tilde{z}, t) \mathrm{d} \tilde{z} \\
& =\pi R_{\text {in }}^{2} \int_{-\infty}^{+\infty} \hat{\mathfrak{h}}_{\alpha}(z-\tilde{z}) \psi_{\alpha}^{z}(\tilde{z}, t) \mathrm{d} \tilde{z} \\
& =\pi R_{\text {in }}^{2}\left[\hat{\mathfrak{h}}_{\alpha} * \psi_{\alpha}^{z}(\cdot, t)\right](z),
\end{aligned}
$$

thereby expressing $y_{\alpha}^{z}(z, t)$ as the convolution product between $\hat{\mathfrak{h}}_{\alpha}$ and $\psi_{\alpha}^{z}(\cdot, t)$.

- Compute the Fourier transform of $y_{\alpha}^{z}(z, t)$ as written in Equation (45), i.e.,

$$
\begin{aligned}
\mathscr{F}\left[y_{\alpha}^{z}(\cdot, t)\right](\xi) & :=\int_{-\infty}^{+\infty} y_{\alpha}^{z}(z, t) \exp (-\mathrm{i} \xi z) \mathrm{d} z \\
& =\pi R_{\text {in }}^{2} \mathscr{F}\left[\hat{\mathfrak{h}}_{\alpha}\right](\xi) \mathscr{F}\left[\psi_{\alpha}^{z}(\cdot, t)\right](\xi) \\
& =\pi R_{\text {in }}^{2} \frac{2 \Gamma(1-\alpha)}{\mathcal{N}(\alpha)} \sin \left(\frac{\alpha \pi}{2}\right)|\xi|^{\alpha-1} \mathscr{F}\left[\psi_{\alpha}^{z}(\cdot, t)\right](\xi),
\end{aligned}
$$

where $\xi \in \mathbb{R} \backslash\{0\}$ is the wave number, $\Gamma(\cdot)$ is the Euler Gamma function and we used the Fourier transform of $\hat{\mathfrak{h}}_{\alpha}$, i.e.,

$$
\mathscr{F}\left[\hat{\mathfrak{h}}_{\alpha}\right](\xi)=\frac{2 \Gamma(1-\alpha)}{\mathcal{N}(\alpha)} \sin \left(\frac{\alpha \pi}{2}\right)|\xi|^{\alpha-1} .
$$

Since $\mathscr{F}\left[y_{\alpha}^{z}(\cdot, t)\right](\xi)$ is proportional to the product of $\mathscr{F}\left[\hat{\mathfrak{h}}_{\alpha}\right](\xi)$ and $\mathscr{F}\left[\psi_{\alpha}^{z}(\cdot, t)\right](\xi)$, one can identify the non-local contribution of the mass flux with $\mathscr{F}\left[\hat{\mathfrak{h}}_{\alpha}\right](\xi)$, given in Equation (47).

Note that, if $\mathfrak{d}_{\alpha}(z, t)$ and $c_{\mathrm{a}}(z, t)$ are both assumed to be even with respect to $z=0$-an assumption that is consistent with the hypothesis, done later, that the considered problem is symmetric with respect to $z=0-, \mathscr{F}\left[y_{\alpha}^{z}(\cdot, t)\right](\xi)$ can be prolonged to $\xi=0$ and is null for this value. To see this, we first rewrite $\mathscr{F}\left[\psi_{\alpha}^{z}(\cdot, t)\right](\xi)$ as

$$
\mathscr{F}\left[\psi_{\alpha}^{z}(\cdot, t)\right](\xi)=-\varrho_{\mathrm{f}} \int_{-\infty}^{+\infty} \mathfrak{d}_{\alpha}(z, t) \partial_{z} c_{\mathrm{a}}(z, t) \exp (-\mathrm{i} \xi z) \mathrm{d} z
$$

Then, we notice that $\mathscr{F}\left[\psi_{\alpha}^{z}(\cdot, t)\right](0)$ is zero, because $\mathfrak{d}_{\alpha}(z, t)$ is even and $\partial_{z} c_{\mathrm{a}}(z, t)$ is odd with respect to $z=0$ for all times. Moreover, because of this result, it also holds that $\lim _{\xi \rightarrow 0}|\xi|^{\alpha-1} \mathscr{F}\left[\psi_{\alpha}^{z}(\cdot, t)\right](\xi)=0$, and, consequently, $\lim _{\xi \rightarrow 0} \mathscr{F}\left[y_{\alpha}^{z}(\cdot, t)\right](\xi)=0$ too. 
- Compute the limit of $\mathscr{F}\left[y_{\alpha}^{z}(\cdot, t)\right](\xi)$ for $\alpha \rightarrow 1^{-}$, and find $\mathcal{N}(\alpha)$ such that

$$
\begin{aligned}
\lim _{\alpha \rightarrow 1^{-}} \mathscr{F}\left[y_{\alpha}^{z}(\cdot, t)\right](\xi) & =\lim _{\alpha \rightarrow 1^{-}} \mathscr{F}\left[\psi_{\alpha}^{z}(\cdot, t)\right](\xi) \\
& =\mathscr{F}\left[-\varrho_{\mathrm{f}} \mathfrak{d}_{1}(\cdot, t) \partial_{z} c_{\mathrm{a}}(\cdot, t)\right](\xi),
\end{aligned}
$$

with $\mathfrak{d}_{1}(\tilde{z}, t):=\lim _{\alpha \rightarrow 1^{-}} \mathfrak{d}_{\alpha}(\tilde{z}, t)$. We emphasise that this limit is taken uniformly with respect to the pairs $(\tilde{z}, t)$ and, in particular, looking at Equation (24), it turns out to be uniform with respect to the motion, so that it is intended as

$$
\begin{aligned}
\lim _{\alpha \rightarrow 1^{-}} \mathfrak{d}_{\alpha}(\tilde{z}, t) & =\lim _{\alpha \rightarrow 1^{-}} \mathfrak{d}_{\alpha}\left(\chi^{z}(\tilde{X}, t), t\right)=\frac{J(\tilde{X}, t)-J_{\gamma}(\tilde{X}, t) \Phi_{\mathrm{s} \nu}}{J(\tilde{X}, t)} \lim _{\alpha \rightarrow 1^{-}} \mathfrak{d}_{\mathrm{R} \alpha} \\
& =\frac{J(\tilde{X}, t)-J_{\gamma}(\tilde{X}, t) \Phi_{\mathrm{s} \nu}}{J(\tilde{X}, t)} \mathfrak{d}_{\mathrm{R} 1},
\end{aligned}
$$

where, in our model, $\mathfrak{d}_{\mathrm{R} 1}$ is a constant having the physical dimensions of a standard diffusivity coefficient. In particular, to meet this requirement, we choose $\mathfrak{d}_{\mathrm{R} \alpha}$ as

$$
\mathfrak{d}_{\mathrm{R} \alpha}:=d_{\mathrm{R}} L^{\alpha-1},
$$

with $d_{\mathrm{R}}$ being a constant reference value for the standard diffusivity coefficient [13], so that $\mathfrak{d}_{\mathrm{R} 1}=d_{\mathrm{R}}$.

One possible way to comply with Equation $(49)$ is that $\mathcal{N}(\alpha)$ satisfies the relation

$$
\lim _{\alpha \rightarrow 1^{-}} \frac{2 \Gamma(1-\alpha) \pi R_{\text {in }}^{2}}{\mathcal{N}(\alpha)}=1 .
$$

Then, for Equation (44) to be (up to the diffusivity $\mathfrak{d}_{\alpha}$ ) Caputo's symmetrised fractional derivative of the mass fraction, $c_{\mathrm{a}}$, which is defined over the interval $]-\ell(t),+\ell(t)[$, we choose the stronger condition

$$
\left.\mathcal{N}(\alpha)=2 \Gamma(1-\alpha) \pi R_{\text {in }}^{2}, \quad \alpha \in\right] 0,1[.
$$

Clearly, Equation (53) represents a "guess", because we are unable to compute directly the normalisation factor for a bounded interval. Nevertheless, plugging Equation (53) into Equation (44) yields

$$
y_{\alpha}^{z}(z, t)=-\frac{\varrho_{\mathrm{f}}}{2 \Gamma(1-\alpha)} \int_{-\ell(t)}^{+\ell(t)} \frac{1}{|z-\tilde{z}|^{\alpha}} \mathfrak{d}_{\alpha}(\tilde{z}, t) \partial_{\tilde{z}} c_{\mathrm{a}}(\tilde{z}, t) \mathrm{d} \tilde{z}
$$

which, apart from the spatial dependence of the fractional diffusivity $\mathfrak{d}_{\alpha}(\tilde{z}, t)$, coincides with the definition of fractional mass flux in one dimension used by other Authors, see for instance [89, 35] and the references therein. Furthermore, in the case in which the fractional diffusivity can be factorised outside the integral operator, e.g. by setting $\mathfrak{d}_{\alpha}(\tilde{z}, t)=\mathfrak{d}_{0 \alpha}$, the axial mass flux becomes proportional to the symmetrised Caputo fractional derivative of order $\alpha$ of $c_{\mathrm{a}}[9]$. 
Remark 3 ((On the normalisation factor $))$ We notice that, apart from the presence of the area of the cylinder's cross-section $\left|\mathscr{C}_{\mathrm{R}}\right|=\pi R_{\mathrm{in}}^{2}$, the expression of the normalisation factor $\mathcal{N}(\alpha)$ given in Equation (53) coincides with the one used in other works (see e.g. [112, 11, [22]). Nevertheless, by looking at Equation (46), one can see that other definitions of the normalisation factor can be employed which satisfy the condition of Equation (49). Indeed, if the limit in Equation (52) is rephrased as

$$
\lim _{\alpha \rightarrow 1^{-}} \frac{2 \Gamma(1-\alpha) \sin (\alpha \pi / 2) \pi R_{\text {in }}^{2}}{\hat{\mathcal{N}}(\alpha)}=1
$$

where $\hat{\mathcal{N}}(\alpha)$ is the new normalisation factor sought for, then, upon following the reasoning leading to Equation (53), one can take $\hat{\mathcal{N}}(\alpha)$ as

$$
\hat{\mathcal{N}}(\alpha):=2 \Gamma(1-\alpha) \sin (\alpha \pi / 2) \pi R_{\text {in }}^{2},
$$

thereby automatically satisfying Equation (55). Then, by using $\hat{\mathcal{N}}(\alpha)$ in Equation (44) in lieu of $\mathcal{N}(\alpha)$, the axial mass flux can be written as

$$
\begin{aligned}
\hat{y}_{\alpha}^{z}(z, t) & =-\frac{\varrho_{\mathrm{f}}}{2 \Gamma(1-\alpha) \sin (\alpha \pi / 2)} \int_{-\ell(t)}^{+\ell(t)} \frac{1}{|z-\tilde{z}|^{\alpha}} \mathfrak{d}_{\alpha}(\tilde{z}, t) \partial_{\tilde{z}} c_{\mathrm{a}}(\tilde{z}, t) \mathrm{d} \tilde{z} \\
& =\mathscr{I}_{-\ell(t),+\ell(t)}^{1-\alpha}\left[-\varrho_{\mathrm{f}} \mathfrak{d}_{\alpha} \partial_{\tilde{z}} c_{\mathrm{a}}\right](z, t),
\end{aligned}
$$

where $\mathscr{I}_{-\ell(t),+\ell(t)}^{1-\alpha}\left[-\varrho_{\mathrm{f}} \mathfrak{d}_{\alpha} \partial_{\tilde{z}} c_{\mathrm{a}}\right]$ is the one-dimensional Riesz potential of $-\varrho_{\mathrm{f}} \mathfrak{d}_{\alpha} \partial_{\tilde{z}} c_{\mathrm{a}}$, but with integration limits $\pm \ell(t)$ instead of $\pm \infty$ (see [104] page 223). For this reason, one may refer to Equation (57) as a "truncated" Riesz potential [38].

At this point, two comments are in order. First, we note that, for $\alpha \rightarrow 1^{-}$, both choices of the normalisation factor lead to the same result and, consequently, the mass flux obtained for $\alpha \rightarrow 1^{-}$ is the same in both formulations. However, something different occurs for $\alpha \rightarrow 0^{+}$. Indeed, by looking at Equation (46), if the normalisation factor $\mathcal{N}(\alpha)$ is used, we obtain, for $\xi \neq 0$, that

$$
\lim _{\alpha \rightarrow 0^{+}} \mathscr{F}\left[y_{\alpha}^{z}(\cdot, t)\right](\xi)=0,
$$

which suggests that the flux of the species is null for $\alpha \rightarrow 0^{+}$. On the contrary, if in Equation (46) $\mathcal{N}(\alpha)$ is replaced with $\hat{\mathcal{N}}(\alpha)$, one obtains, for $\xi \neq 0$,

$$
\lim _{\alpha \rightarrow 0^{+}} \mathscr{F}\left[\hat{y}_{\alpha}^{z}(\cdot, t)\right](\xi)=|\xi|^{-1} \mathscr{F}\left[-\varrho_{\mathrm{f}} \mathfrak{d}_{0}(\cdot, t) \partial_{z} c_{\mathrm{a}}(\cdot, t)\right](\xi),
$$

with $\mathfrak{d}_{0}=\lim _{\alpha \rightarrow 0^{+}} \mathfrak{d}_{\alpha}$, thereby implying, in general, a non-zero flux. In view of the above results and of the normalisation factor used by other Authors [89, 35, 11, 105], we prefer to employ $\mathcal{N}(\alpha)$ as normalisation factor in the remainder of this work. Besides, in this way, the model is able to account for a wider range of diffusion situations, from no diffusion to standard diffusion. Nevertheless, for completeness in our study, in Section "Results and discussion", we provide a comparison between the approach involving $\mathcal{N}(\alpha)$ and that involving $\hat{\mathcal{N}}(\alpha)$.

Now, the restrictions imposed on the motion imply that the only component of interest of the deformation gradient tensor is given by $[\boldsymbol{F}(X, t)]^{z} Z=1+u^{\prime}(Z, t)$. Thus, by taking into account Equation (25), the material fractional diffusivity tensor can be rephrased as follows

$$
\left[\mathfrak{D}_{\alpha}(X, \tilde{X}, t)\right]^{Z Z}=\mathfrak{d}_{\mathrm{R} \alpha} \frac{1+u^{\prime}(\tilde{Z}, t)-J_{\gamma}(\tilde{Z}, t) \Phi_{\mathrm{s} \nu}}{\left[1+u^{\prime}(Z, t)\right]\left[1+u^{\prime}(\tilde{Z}, t)\right]},
$$


whereas the definition (43) implies that $\mathfrak{F}_{\alpha}$, given in Equation (22b), can be rephrased as a function of $Z, \tilde{Z}$ and $t$, i.e.,

$$
\left.\mathfrak{F}_{\alpha}(X, \tilde{X}, t)=\mathfrak{H}_{\alpha}(Z, \tilde{Z}, t)=\frac{1}{2 \Gamma(1-\alpha) \pi R_{\mathrm{in}}^{2}} \frac{1}{|Z+u(Z, t)-\tilde{Z}-u(\tilde{Z}, t)|^{\alpha}}, \quad \alpha \in\right] 0,1[.
$$

Finally, by substituting Equation (60) into Equation (23b), and taking into account relation (22b), the only non-zero component of the material fractional mass flux vector, $\boldsymbol{Y}_{\alpha}$, is the one along the axial direction, and represents the backward Piola transform of Equation (44), i.e.,

$$
Y_{\alpha}^{Z}(Z, t)=-\frac{\varrho_{\mathrm{f}}}{2 \Gamma(1-\alpha)} \int_{-L_{\mathrm{in}}}^{+L_{\mathrm{in}}} \mathfrak{d}_{\mathrm{R} \alpha} \frac{\left[1+u^{\prime}(\tilde{Z}, t)-J_{\gamma}(\tilde{Z}, t) \Phi_{\mathrm{s} \nu}\right]}{|Z+u(Z, t)-\tilde{Z}-u(\tilde{Z}, t)|^{\alpha}} \frac{\mathfrak{c}_{\mathrm{a}}^{\prime}(\tilde{Z}, t)}{\left[1+u^{\prime}(\tilde{Z}, t)\right]} \mathrm{d} \tilde{Z} .
$$

Looking at Equations (61) and (62), we remark that, in contrast to what is usually assumed in the "standard" setting of Fractional Calculus, both $\mathfrak{H}_{\alpha}$ and $Y_{\alpha}^{Z}$ depend on the displacement field, rather than depending on the difference between $Z$ and $\tilde{Z}$, only. As anticipated in the Introduction, this result is one of the most relevant novelties of our work, as it prescribes that the non-locality evolves with the change of configuration of the system. Moreover, since in our framework the displacement is driven by growth (even though $u$ and $\gamma$ are formally independent variables), we conclude that the non-locality of the problem is related also to the variation of the tissue's internal structure, as modelled by $\gamma$.

\section{Results and discussion}

In this section, we study the impact of the non-local diffusion of nutrients on the benchmark problem specified above. For this scope, we distinguish between two mathematical models, both characterised by Equations (26a)-(26e). The first model, referred to as fractional model, describes the growth of the considered avascular tumour in the case in which the diffusion of the nutrients is governed by the non-local constitutive law (62). The second model, denominated standard model, describes the growth of the tumour by employing the same governing equations (26a)-(26e), with the only difference being that the nutrients' diffusive mass flux vector is expressed by standard Fick's law, i.e.,

$$
\boldsymbol{Y}_{\text {std }}(X, t)=-\varrho_{\mathrm{f}} \boldsymbol{D}(X, t) \operatorname{Gradc} \mathfrak{c}_{\mathrm{a}}(X, t),
$$

where "std" stands for "standard", and $\boldsymbol{D}$ is the material diffusivity tensor, given by [101, 56

$$
\boldsymbol{D}(X, t)=\left(J(X, t)-J_{\gamma}(X, t) \Phi_{\mathrm{s} \nu}\right) d_{\mathrm{R}} \boldsymbol{C}^{-1}(X, t) .
$$

We notice that both models, i.e., the fractional and the standard one, share the same set of parameters except for the reference diffusivities $\mathfrak{d}_{\mathrm{R} \alpha}$ and $d_{\mathrm{R}}$. Note also that Equation (64) can be obtained from (25) by setting $\tilde{X}=X$ and then taking the limit for $\alpha \rightarrow 1^{-}$, i.e., $\lim _{\alpha \rightarrow 1^{-}} \mathfrak{D}_{\alpha}(X, X, t)=$ $D(X, t)$.

For the purposes of our work, one should not fix $\mathfrak{d}_{\mathrm{R} \alpha}$ independently of $d_{\mathrm{R}}$. Indeed, in order to compare the results of the non-local model with those of the local one, $\mathfrak{d}_{\mathrm{R} \alpha}$ must depend on $d_{\mathrm{R}}$ in such a way that it tends to $d_{\mathrm{R}}$ in the limit $\alpha \rightarrow 1^{-}$. For this reason, and taking into account 
that there exist several experimental works in which the standard diffusivity of species in biological tissues has been measured (see e.g. [62, [59]), we use for $\mathfrak{d}_{\mathrm{R} \alpha}$ the definition given in Equation (51), and we set $L=2 L_{\text {in }}$. In Table 1, we provide the list of all the parameters used in our simulations. We remark that, due to the symmetries of the benchmark problem studied in this work, in the following we report the profile of the main quantities of interest restricted to half of the domain, i.e., $\left[0, L_{\text {in }}\right]$.

Table 1: List of parameters used in the numerical simulations.

\begin{tabular}{|c|c|c|c|c|}
\hline Parameter & Unit & Value & Equation & Reference \\
\hline$L_{\text {in }}$ & $\mathrm{cm}$ & 0.500 & (44) & 101 \\
\hline$R_{\text {in }}$ & $\mathrm{cm}$ & $1.000 \cdot 10^{-2}$ & 62 & [101] \\
\hline$\lambda$ & $\mathrm{Pa}$ & $1.333 \cdot 10^{4}$ & 12 & [111] \\
\hline$\mu$ & $\mathrm{Pa}$ & $1.999 \cdot 10^{4}$ & 12 & [111] \\
\hline$k_{\mathrm{R}}$ & $\mathrm{m}^{2} /(\mathrm{Pas})$ & $4.875 \cdot 10^{-13}$ & 15 & 62 \\
\hline$m_{0}$ & - & 0.0848 & 15 & 62 \\
\hline$m_{1}$ & - & 4.638 & 15 & 62 \\
\hline$d_{\mathrm{R}}$ & $\mathrm{m}^{2} / \mathrm{s}$ & $3.200 \cdot 10^{-9}$ & 51 & [107] \\
\hline$\zeta_{\text {fp }}$ & $\mathrm{kg} /\left(\mathrm{m}^{3} \mathrm{~s}\right)$ & $1.343 \cdot 10^{-3}$ & $27 \mathrm{a}$ & [25] \\
\hline$\zeta_{\mathrm{nf}}$ & $\mathrm{kg} /\left(\mathrm{m}^{3} \mathrm{~s}\right)$ & $1.150 \cdot 10^{-5}$ & (27b) & [25] \\
\hline$\zeta_{\mathrm{cp}}$ & $\mathrm{kg} /\left(\mathrm{m}^{3} \mathrm{~s}\right)$ & $3.000 \cdot 10^{-4}$ & $27 \mathrm{c}$ & [23, 24] \\
\hline$\zeta_{\mathrm{pn}}$ & $\mathrm{kg} /\left(\mathrm{m}^{3} \mathrm{~s}\right)$ & $1.500 \cdot 10^{-3}$ & $27 \mathrm{~d}$ & [25] \\
\hline $\mathfrak{c}_{\mathrm{cr}}$ & - & $1.000 \cdot 10^{-3}$ & $27 \mathrm{a}$ & [101] \\
\hline $\mathfrak{c}_{\mathrm{env}}$ & - & $7.000 \cdot 10^{-3}$ & $27 \mathrm{a}$ & [101] \\
\hline $\mathfrak{c}_{0}$ & - & $1.000 \cdot 10^{-2}$ & $27 \mathrm{c}$ & This work \\
\hline$\delta_{1}$ & - & $7.138 \cdot 10^{-1}$ & $27 a$ & 80 \\
\hline$\delta_{2}$ & $\mathrm{~Pa}$ & $1.541 \cdot 10^{3}$ & $27 \mathrm{a}$ & [80] \\
\hline$\Phi_{\mathrm{S} \nu}$ & - & 0.8 & (5a) & [101] \\
\hline$\varrho_{\mathrm{s}}$ & $\mathrm{kg} / \mathrm{m}^{3}$ & 1000 & $(2)$ & [101] \\
\hline$\varrho_{\mathrm{f}}$ & $\mathrm{kg} / \mathrm{m}^{3}$ & 1000 & (2) & [101] \\
\hline
\end{tabular}

To start with, in Fig. 1. we report the spatial profile of the nutrients' mass fraction $\mathfrak{c}_{\mathrm{a}}(Z, t)$. Specifically, in the left panel of Fig. 1. we present the results of our simulations for $\alpha=0.1$ (dashed line) and $\alpha=0.9$ (solid line), and for different times. As shown in this plot, the parameter $\alpha$ permits to control how the nutrients diffuse into the tumour from the axial boundaries (i.e., the terminal cross sections $Z= \pm L_{\text {in }}$ ). In particular, for $\alpha=0.1$ the diffusion of the nutrients is constrained to the tumour's axial boundary, i.e., close to $Z= \pm L_{\text {in }}$, so that their mass fraction is dramatically reduced in the internal points of the specimen. In such a situation, the proliferating cells consume the nutrients that are already present in the tissue, without the replenishment needed to continue their proliferation. On the contrary, for $\alpha=0.9$, the nutrients are able to diffuse towards the centre of the tumour, so that their consumption is less localised. For clarity, in the plot we prefer to show only the curves corresponding to $\alpha=0.1$ and $\alpha=0.9$. For any other value of $\alpha \in] 0.1,0.9[$, the model is able to describe different diffusion profiles ranging between the ones obtained for $\alpha=0.1$ and for $\alpha=0.9$. To us, an interesting feature of the curves corresponding to $\alpha=0.1$ is that, depending on the point $Z$ at which the nutrients' mass fraction is observed, the trend of these 
curves exhibits a different monotonicity in time. Indeed, the nutrients' mass fraction decreases in time close to the boundary $Z=L_{\text {in }}$, whereas it increases towards the tumour's centre. Furthermore, in the panel on the right of Fig. 1. we compare, for different values of $\alpha$, the results obtained with the fractional model with those obtained with the standard model at time $t=20 \mathrm{~d}$. Specifically, for $\alpha$ close to 0 , there is almost no diffusion, while, when $\alpha$ is close to 1 , the fractional model conducts to the standard one, as evidenced by our previous calculations (see Equation (46)).
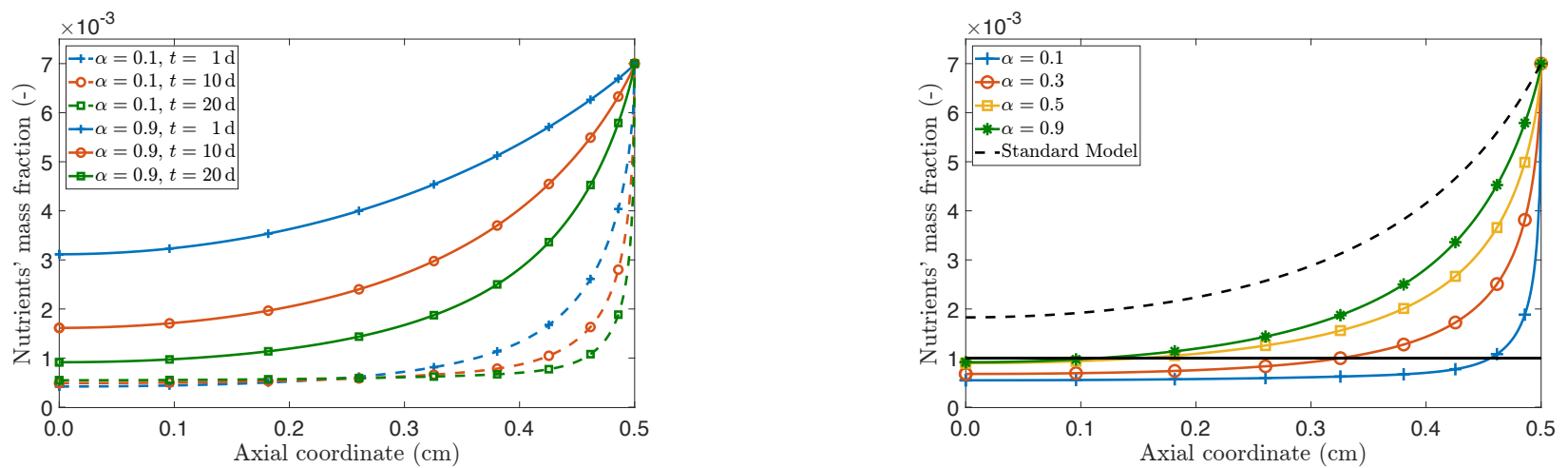

Figure 1: Spatial profile of the nutrients' mass fraction $\mathfrak{c}_{\mathrm{a}}(Z, t)$ for different values of $\alpha$ and at different times (panel on the left), and comparison of the results obtained with the fractional and the standard model at time $t=20 \mathrm{~d}$ (panel on the right).

As shown in Fig. 2, the non-local way in which the nutrients diffuse into the tissue affects the manner in which the tumour grows. By increasing $\alpha$ and, thus, enhancing diffusion, one also increases the availability of the nutrients in the tumour, thereby boosting its growth. On the other hand, for $\alpha=0.1$, the displacement is hindered and its highest values are attained in a neighbourhood of $Z=L_{\text {in }}$. Indeed, this is where the nutrients enter the tumour and their mass fraction still remains high enough to trigger growth, so that the magnitude of the displacement in this region of the tumour is higher than elsewhere. However, moving towards the interior of the tumour, the fact that the nutrients' concentration is below the critical threshold brings growth to a stop, thereby considerably reducing the magnitude of the displacement. This behaviour shows that also the monotonicity in time of the displacement curves depends on the point $Z$ at which they are reckoned. More in detail, the reduction of the displacement in the interior of the tumour may be due to the loss of mass caused by the lack of nutrients, which implies that the proliferating cells start to die, and a region of necrotic cells comes into sight. This behaviour becomes even more evident by looking at the left panel of Fig. 3. Moreover, comparing the right panels of Fig. 1 and Fig. 3, we notice that the part of the domain in which the necrotic cells appear coincides with the one in which the nutrients fall below the critical value $\mathfrak{c}_{\mathrm{cr}}$, represented with the solid horizontal line in the right panel of Fig. 1. By referring to Equation $(27 \mathrm{~d})$, when $\mathfrak{c}_{\mathrm{a}}<\mathfrak{c}_{\mathrm{cr}}$, the rate of mass $R_{\text {pn }}$ becomes active and, therefore, the proliferating cells change into necrotic cells.

To continue our analysis, we refer to Fig. 4. where we plot the growth parameter $\gamma$. By focusing on the panel on the left, we notice, for $\alpha=0.1$, a localisation of the variation of the growth parameter near the boundary $Z=L_{\text {in }}$ for increasing time, whereas, for $\alpha=0.9$, the variation of $\gamma$ is more uniformly distributed in the whole domain. Besides, for $\alpha=0.1, \gamma$ is greater than one for all $Z \in\left[0, L_{\mathrm{in}}\right]$ and for all $t$, even though this is difficult to be observed with the unaided eye. 

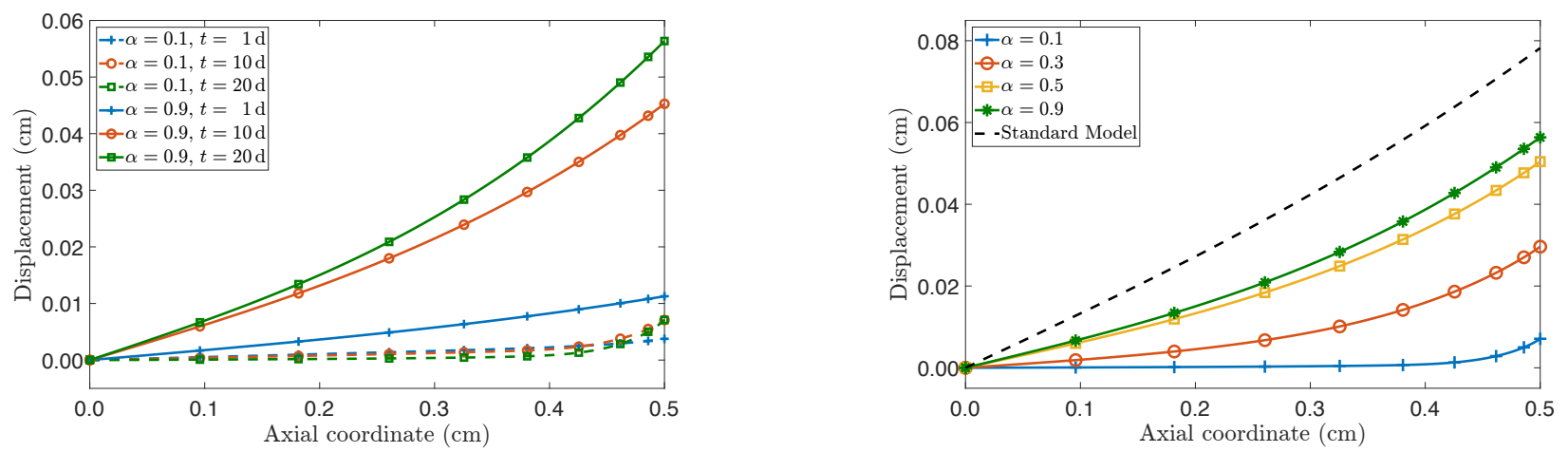

Figure 2: Spatial profile of the axial displacement $u(Z, t)$ for different values of $\alpha$ and at different times (panel on the left), and comparison of the results obtained with the fractional and the standard model at time $t=20 \mathrm{~d}$ (panel on the right).
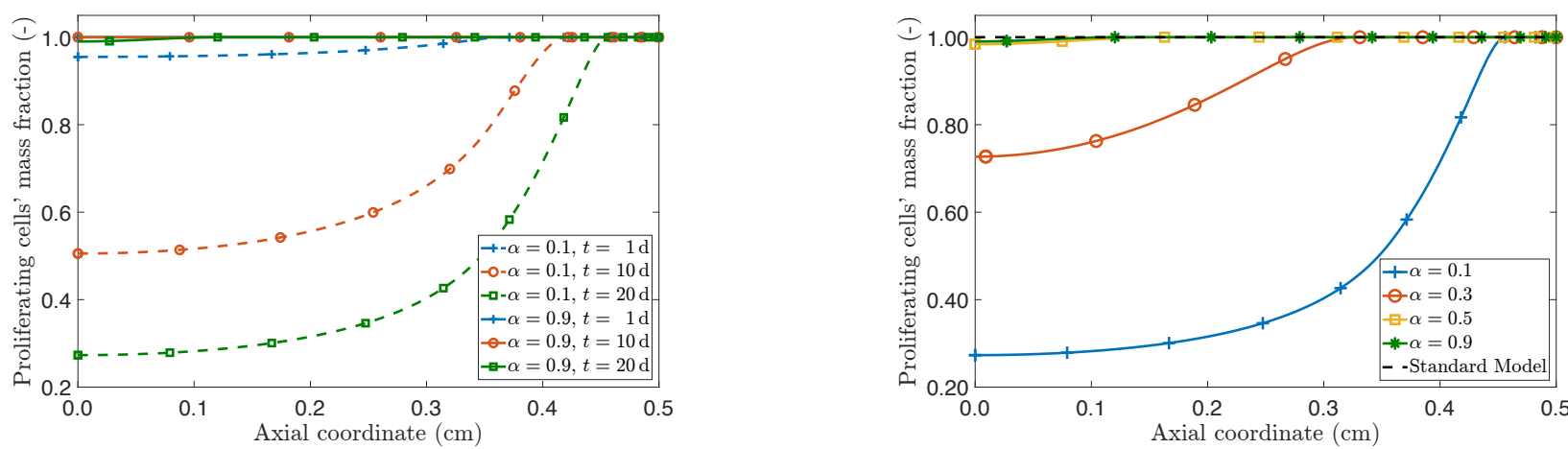

Figure 3: Spatial profile of the proliferating cells' mass fraction $\mathfrak{c}_{\mathrm{p}}(Z, t)$ for different values of $\alpha$ and at different times (panel on the left), and comparison of the results obtained with the fractional and the standard model at time $t=20 \mathrm{~d}$ (panel on the right).

This is because, although for $t \geq 1 \mathrm{~d}$ the mass fraction of the nutrients is above the threshold value $\mathfrak{c}_{\mathrm{cr}}$ mostly near the boundary (see the left panel of Fig. 1), the inner region has undergone a growth process at earlier times. Indeed, since the condition $\mathfrak{c}_{\mathrm{a}}(Z, 0) \equiv \mathfrak{c}_{\mathrm{env}}>\mathfrak{c}_{\mathrm{cr}}$ is respected, the mass rate $R_{\mathrm{fp}}$ is greater than zero, and we can conclude that, from the very beginning, the cell proliferation is promoted until the nutrients' concentration falls below its critical value. Note also that this is accelerated when $\alpha$ is near zero because of the slow pace with which the nutrients are refilled. At this point, the proliferating cells abruptly die, thereby turning into necrotic cells, and go into the fluid (see the definition of $R_{\mathrm{nf}}$ ), which results in a loss of mass. For $\alpha=0.9$, instead, it is visible also with the naked eye that $\gamma$ is greater than unity everywhere in $\left[0, L_{\text {in }}\right]$ and for all the considered times. Finally, as noticed for the nutrients' mass fraction and for the displacement, also the monotonicity in time of the trend of the growth parameter depends, for $\alpha=0.1$, on the point $Z$ at which $\gamma$ is observed. Indeed, $\gamma$ is monotonically increasing in time for $Z$ close to $Z=L_{\text {in }}$, and monotonically decreasing for $Z$ "moving" towards the centre of the tumour.

Now, we report the evolution of the pressure, $\mathfrak{p}$, in Fig. 5. For both the standard and the fractional model, when $\alpha$ is close to 1, the pressure of the interstitial fluid decreases, taking negative 

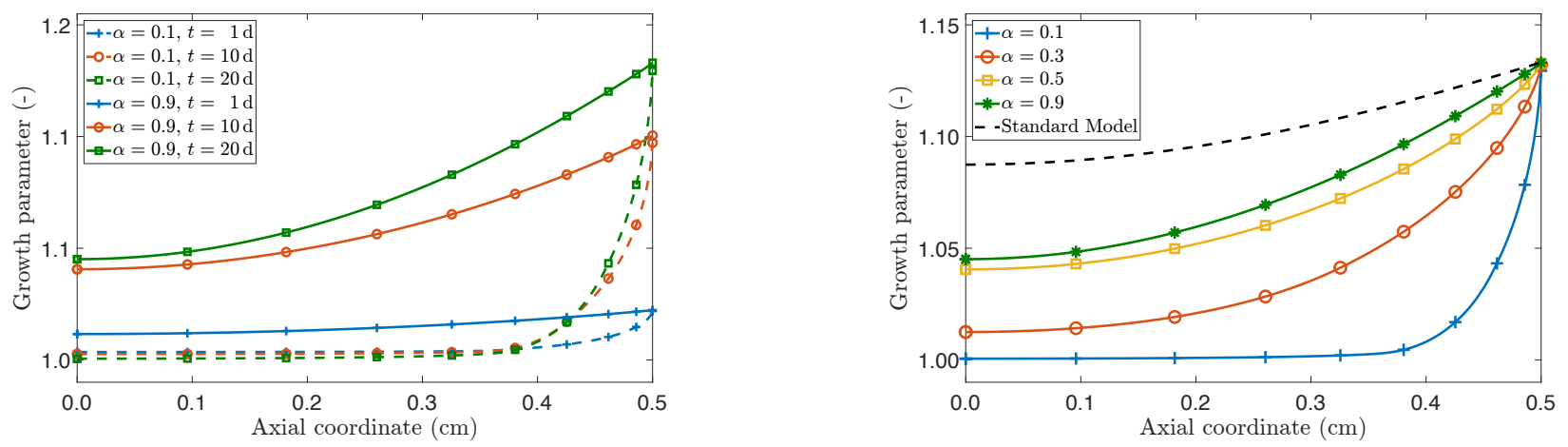

Figure 4: Spatial profile of the growth parameter $\gamma(Z, t)$ for different values of $\alpha$ and at different times (panel on the left), and comparison of the results obtained with the fractional and the standard model at time $t=20 \mathrm{~d}$ (panel on the right).
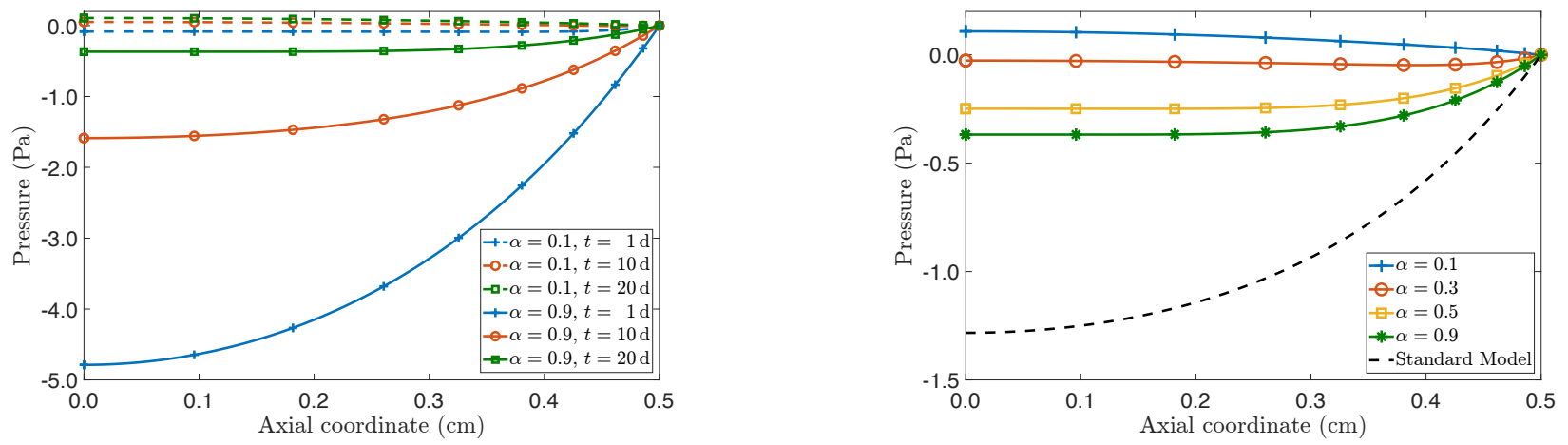

Figure 5: Spatial profile of the pressure $\mathfrak{p}(Z, t)$ for different values of $\alpha$ and at different times (panel on the left), and comparison of the results obtained by the fractional and the standard model at time $t=20 \mathrm{~d}$ (panel on the right).

values, from the free boundary towards the tumour's centre. However, for $\alpha$ tending towards 0 from above, the pressure in the interior of the tumour tends to become positive. To explain this event, we notice that the proliferating cells absorb fluid from the surrounding environment to fuel their growth, which is possible because the fluid flows towards the tumour's interior. However, due to an over-consumption of nutrients, the level of those drastically decreases in the innermost zone of the tumour. This situation, as evidenced in our simulations (see Fig. 4), creates a layer of proliferating cells near the outer surface (i.e., the cross section $Z=L_{\text {in }}$ ), and a region of necrotic cells at the centre of the tumour. By looking at Equation (27b), in this circumstance, the necrotic cells dissolve into the fluid with rate $\zeta_{\text {nf }}$, thereby increasing its pressure, which, in turn, generates an outward flux (i.e., a flux in the direction opposite to the fluid flow). This sequence of events, which are consistent with the biological foundations of nutrient diffusion and necrosis in a tumour as explained in 77], arises in the model thanks to the non-local approach presented in this work. That is, the non-locality parameter $\alpha$ is responsible for this picture and, thus, through its inclusion, the fractional model is able to reproduce a scenario that was not initially considered in the model. On the contrary, as the results show, this behaviour would not be observed within a formulation 
based on standard Fick's law, at least with our model as is.

Finally, as we mentioned before (see Remark 3), for completeness in our discussion, we compare the results corresponding to the adoption of $\mathcal{N}(\alpha)$ versus those obtained with $\hat{\mathcal{N}}(\alpha)$. As shown in Fig. 6. top left panel, when the normalisation factor is $\hat{\mathcal{N}}(\alpha)$, we observe, for $\alpha \rightarrow 0^{+}$, a less pronounced decrease of the nutrients' mass fraction. This is compatible with the fact that, even for very small values of $\alpha$, there is an incoming mass flux of nutrients through the domain's boundaries that reestablishes the nutrients eaten by the cells. This effect, in turn, tends to disappear when the normalisation factor $\mathcal{N}(\alpha)$ is employed since, in that case, the mass flux tends to zero in the limit $\alpha \rightarrow 0^{+}$. Coherently with this observation, we also notice a markedly different behaviour of the growth parameter (see Fig. 6, top right panel). Indeed, since the flux of nutrients obtained for $\hat{\mathcal{N}}(\alpha)$ does not vanish for $\alpha \rightarrow 0^{+}$, and a greater amount of nutrients remains available even at time $t=20 \mathrm{~d}$, growth can still occur, as is testified by the dotted line marked with "+". Similar comments pertain also to the description of the displacement (see Fig. 6, bottom left panel). Indeed, since growth remains active also for small values of $\alpha$, the displacement also tends to persist even at $t=20 \mathrm{~d}$, and remains relatively large in the neighbourhood of the domain's boundaries, where the availability of nutrients is the highest (because of the Dirichlet condition assigned to the nutrients' mass fraction) and growth is present. These differences notwithstanding, it should be emphasised that the qualitative behaviour of the curves describing the nutrients' mass fraction and the growth parameter is the same for both choices of the normalisation factor. On the contrary, the behaviour of the pressure (see Fig. 6, bottom right panel) is both qualitatively and quantitatively different for $\alpha=0.1$. In fact, the use of $\hat{\mathcal{N}}(\alpha)$ nullifies the effect visible at $t=20 \mathrm{~d}$, for $\alpha=0.1$ and normalisation factor $\mathcal{N}(\alpha)$, which consisted in the sign change of the pressure. Hence, employing $\hat{\mathcal{N}}(\alpha)$ leaves the pressure negative, thereby triggering no inversion in the flow of the interstitial fluid, which continues to flow from the exterior of the tumour into it.

\section{Conclusions}

In this work, we study the influence of a given type of non-local diffusion of nutrients on the growth of an avascular tumour. For this purpose, we generalise Fick's law of diffusion by introducing a non-local constitutive relationship for the mass flux vector that, after some considerations, can be identified with a fractional derivative of the nutrients' mass fraction. We call attention to the fact that, since we are dealing with growth, we need to describe how the non-locality of the prescribed constitutive law evolves with the deformation and the growth-induced inelastic distortions that accompany the evolution of the system under study. This consideration implies that the nonlocality of the presumed constitutive response should be subordinate to the motion $\chi$ (see Equation (22bD) and, thus, that it cannot depend explicitly on the difference $X-\tilde{X}$ between the reference placements of the material points embedded in $X$ and $\tilde{X}$. Furthermore, we note that, as prescribed by Equation (25), the non-local character of the mass flux vector also depends on the structural changes of the tumour through the determinant of $\boldsymbol{F}_{\gamma}$. To the best of our understanding, the above considerations imply substantial differences between our work and other papers on the subject found in the scientific literature. Moreover, we suggest a formulation of non-local diffusion on manifolds (see Appendix A1).

To investigate the influence of the non-local diffusion of the nutrients on the tumour evolution, we focused on a benchmark problem that allows, due to the enforced symmetries, the reduction 

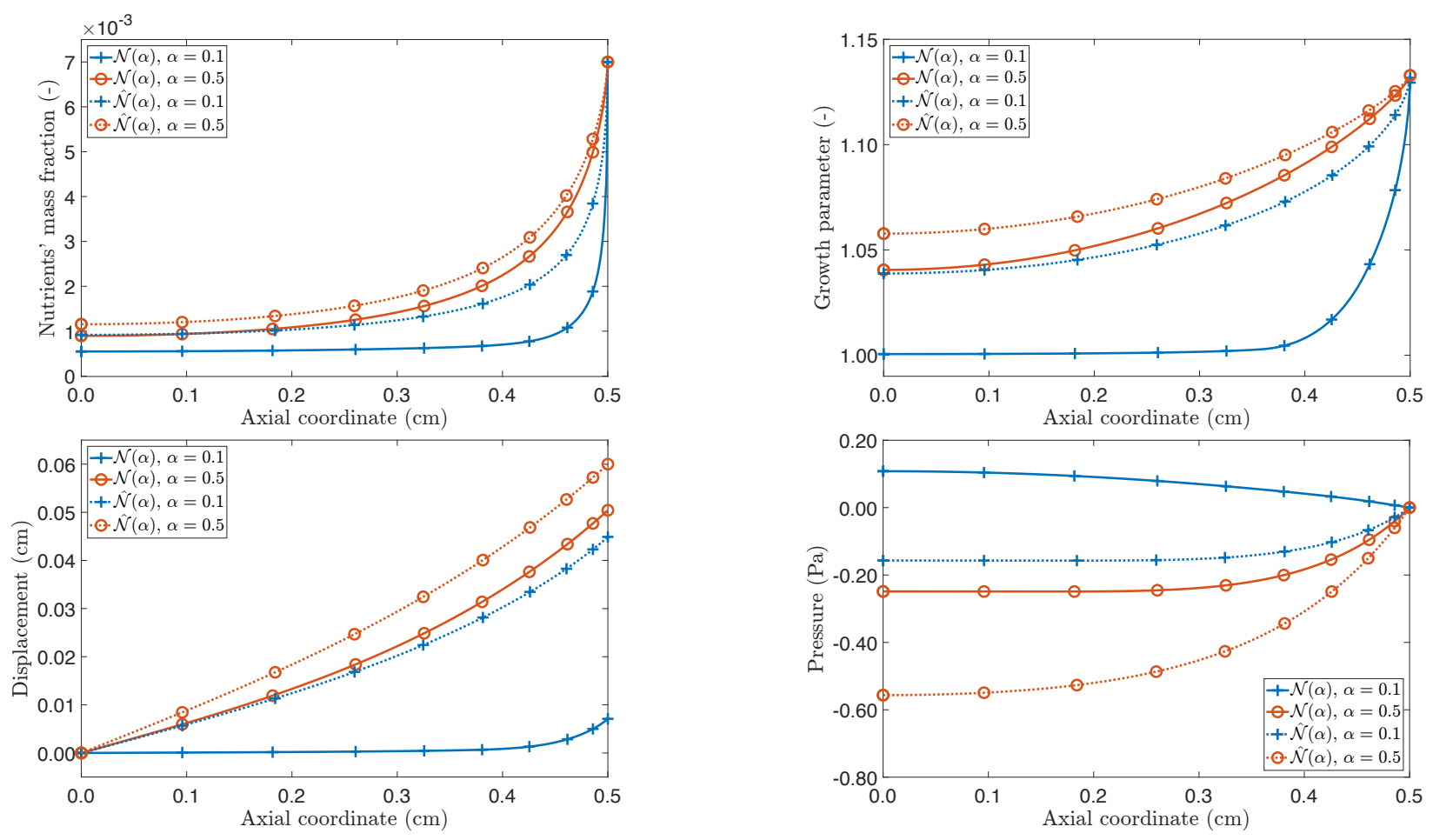

Figure 6: Comparison of the spatial profiles of $\mathfrak{c}_{\mathrm{a}}(Z, t)$ (top left), $\gamma(Z, t)$ (top right), $u(Z, t)$ (bottom left) and $\mathfrak{p}(Z, t)$ (bottom right) for the approaches involving $\mathcal{N}(\alpha)$ (solid line) and $\hat{\mathcal{N}}(\alpha)$ (dotted line). In the plots different values of $\alpha$ are used and time is fixed to $t=20 \mathrm{~d}$.

of the original three-dimensional framework to a one-dimensional problem. This has an important impact on the selection of the non-locality function, $\hat{\mathfrak{f}}_{\alpha}$, which has to be able to capture how the geometrical symmetries of the problem affect the description of the non-locality. Particularly, in our analysis, we re-obtained the definition of one-dimensional fractional mass flux proposed in other works [89, 35].

In our work, the numerical solution of the set of equations defining the mathematical model is found by employing the FE method, which has been adapted for the solution of the fractional diffusion equation (26c). In particular, the obtained numerical results show that the non-local character of the nutrients' evolution has a considerable repercussion on the growth of the hypothetical tumour under study. Specifically, by varying the parameter $\alpha \in] 0,1[$, the model is capable, in the limit cases, of generating situations of no diffusion or of restoring Fick's law. This conclusion evidences the relevance of embracing a fractional framework in our model, since it permits to "control", through the parameter $\alpha$, the way in which the tumour grows. Finally, we discussed a possible way for defining another normalisation factor, termed $\hat{\mathcal{N}}(\alpha)$, involved in the definition of the mass flux vector, and we provided a comparison between the two approaches.

Certainly, our model can be further generalised and, in the following, we discuss some important issues that should be accounted for in forthcoming works. A first issue arises from the fact that, once the dimensionality and the symmetries of the problem at hand are specified, Equation (16) must be adapted accordingly. This implies that the non-locality function and the normalisation 
factors should be conceived in a symmetry- and dimensional-dependent fashion ${ }^{2}$. To find such relations is part of our ongoing research. Additionally, in our model, the information on the microscopic structure of the tumour is not explicitly taken into account and, thus, its contribution is neglected. As pointed out in the Introduction, the multi-scale and heterogeneous character of the environment in which diffusion takes place is one of the main factors influencing the occurrence of non-Fickean diffusion. Therefore, the adoption of mathematical techniques, such as the Asymptotic Homogenisation Method [29, could be capable of incorporating these features into a framework of tissue growth [96] and non-local diffusion.

We further remark that an aspect that is not contemplated in the current formulation of the model is that the chemical agents should be both in the fluid phase and in the solid phase, and not only in the fluid phase. One of the main drawbacks of this phenomenological consideration is that it is not possible to link the mass sources to the chemical potentials of the nutrients, nor is it possible to establish a sound and comprehensive thermodynamic framework accounting for interphase mass transfers as non-equilibrium processes. This implies that no information, or only a limited amount of information, can be extracted from the study of the dissipation inequality of the system (and this is not directly due to the fact that growth necessitates the consideration of processes, of cellular or molecular type, that could not be accounted for in the model). Therefore, under the circumstances of the present model, it is not possible to obtain Equation (16) from the study of the dissipation inequality, as it would be the case in the classical procedure that leads to Fick's law. In this respect, one of the technical difficulties that arise in our work is that we cannot invert the balance of linear momentum associated with the chemical agents, since the inversion of fractional operators is not always permitted. One possible solution, that seems to be thermodynamically acceptable, is to adopt a procedure similar to the one depicted in [58], that is, to consider the part of the dissipation inequality that is of interest for us, to put it in weak form and to express the flux in terms of a non-local constitutive law depending on the gradient of the chemical potential.

Finally, we would like to mention that in recent years Fractional Calculus has demonstrated to be an effective mathematical tool in the description of several phenomena. However, there is still an urgency in incorporating this notion in mathematical models that go beyond the classical ones.

\section{Acknowledgement}

The Authors acknowledge the Dipartimento di Scienze Matematiche (DISMA) "G.L. Lagrange" of the Politecnico di Torino, and that the present research has been partially supported by MIUR grant "Dipartimenti di Eccellenza 2018-2022" ('Departments of Excellence 2018-2022'), project no. E11G18000350001. The Authors warmly thank Prof. Dušan Zorica for his invaluable help, for providing essential references and for the many suggestions that he has given us for this work.

\section{Authors contribution}

All Authors have equally contributed to this work.

\footnotetext{
${ }^{2}$ Similar problems are subject of investigations conducted by our group in conjunction with our colleague Prof. Dušan Zorica (Mathematical Institute, Serbian Academy of Arts and Sciences, Serbia) and started, from our side, during his visit at the Politecnico di Torino (Italy) in January 2020.
} 


\footnotetext{
${ }^{3}$ Given the geodesic from $x$ to $\tilde{x}$, and denoting by $\eta:[0,1] \rightarrow \mathscr{B}_{t}$ its parameterisation, so that $x=\eta(0)$ and $\tilde{x}=\eta(1)$, we set $\operatorname{dist}_{\mathscr{B}_{t}}(x, \tilde{x}):=\int_{0}^{1}\left\|\eta^{\prime}(\sigma)\right\| \mathrm{d} \sigma$.
}

where the non-locality function is given by the following relationship

$$
\mathfrak{f}_{\alpha}(x, \tilde{x}):=\mathfrak{f}_{\alpha}^{(0)}\left(x_{0}, \mathcal{T}_{x}^{x_{0}}(\tilde{x})\right) .
$$

In Equation (66), the notation $\mathcal{T}_{x}^{x_{0}}:=\exp _{x_{0}} \circ\left(\mathcal{P}_{x_{0}}^{x}\right)^{-1} \circ \exp _{x}^{-1}$ is used, and the following operators are introduced:

- Let $T_{x, \delta} \mathscr{B}_{t}$ be the subset of the tangent space $T_{x} \mathscr{B}_{t}$ defined by

$$
T_{x, \delta} \mathscr{B}_{t}:=\left\{\boldsymbol{v}_{x} \in T_{x} \mathscr{B}_{t} \mid\left\langle\boldsymbol{v}_{x}, \boldsymbol{v}_{x}\right\rangle_{\boldsymbol{g}} \leq \delta \text {, with } \delta>0\right\},
$$

and let $\mathscr{U}_{t}(x, \delta):=\left\{\tilde{x} \in \mathscr{B}_{t} \mid \operatorname{dist}_{\mathscr{B}_{t}}(x, \tilde{x}) \leq \delta\right\}$ be a closed neighbourhood of $x$ having radius $\delta$, with dist $\mathscr{B}_{t}: \mathscr{B}_{t} \times \mathscr{B}_{t} \rightarrow \mathbb{R}_{0}^{+}$denoting the distance function ${ }^{3}$ on $\mathscr{B}_{t}$ 106. The operator

$$
\exp _{x}: T_{x, \delta} \mathscr{B}_{t} \rightarrow \mathscr{U}_{t}(x, \delta),
$$

referred to as exponential map, is injective and associates each element of $T_{x, \delta} \mathscr{B}_{t}$ with the point $\tilde{x}=\exp _{x}\left(\boldsymbol{v}_{x}\right) \in \mathscr{U}_{t}(x, \delta)$, which is the projection of $\boldsymbol{v}_{x}$ onto $\mathscr{U}_{t}(x, \delta)$. Note that the result of this operation generalises the concept of translation to the case of a manifold. To construct $\exp _{x}\left(\boldsymbol{v}_{x}\right)$, we take $\boldsymbol{v}_{x} \in T_{x, \delta} \mathscr{B}_{t}$ and consider the unique solution to the geodesic equation (see e.g. [79]), parameterised by $\eta:[0,1] \rightarrow \mathscr{U}_{t}(x, \delta)$, and in harmony with the "initial" conditions $\eta(0)=x$ and $\eta^{\prime}(0)=\boldsymbol{v}_{x}$. Then, we identify $\exp _{x}\left(\boldsymbol{v}_{x}\right)$ with $\eta(1)$, i.e., $\exp _{x}\left(\boldsymbol{v}_{x}\right)=\eta(1) \equiv \tilde{x}$.

By construction, the exponential map is invertible and its inverse, i.e., $\exp _{x}^{-1}: \mathscr{U}_{t}(x, \delta) \rightarrow$ $T_{x, \delta} \mathscr{B}_{t}$, returns a unique tangent vector of $T_{x, \delta} \mathscr{B}_{t}$ for each point of $\mathscr{U}_{t}(x, \delta)$. Therefore, by taking $\tilde{x} \in \mathscr{U}_{t}(x, \delta)$, with $\tilde{x}=\eta(1)$, it holds that $\exp _{x}^{-1}(\eta(1))=\eta^{\prime}(0)$. 
- Let us consider two points of the manifold, e.g. $x_{0}, x \in \mathscr{B}_{t}$, and let $\zeta:[0, s] \rightarrow \mathscr{B}_{t}$, with $\zeta(0)=x_{0}$ and $\zeta(s)=x$, be the parameterisation of the geodesic connecting $x_{0}$ to $x$. Moreover, let us take the sets of tangent vectors $T_{x_{0}, \delta} \mathscr{B}_{t}$ and $T_{x, \delta} \mathscr{B}_{t}$, with $\delta>0$. Then, to transport parallely the elements of $T_{x_{0}, \delta} \mathscr{B}_{t}$ into $T_{x, \delta} \mathscr{B}_{t}$ along the geodesic parameterised by $\zeta$, we define the shifter operator

$$
\mathcal{P}_{x_{0}}^{x}: T_{x_{0}, \delta} \mathscr{B}_{t} \rightarrow T_{x, \delta} \mathscr{B}_{t}, \quad \boldsymbol{v}_{x_{0}} \mapsto \mathcal{P}_{x_{0}}^{x} \boldsymbol{v}_{x_{0}}=\boldsymbol{v}_{x}
$$

Clearly, $\mathcal{P}_{x_{0}}^{x}$ is invertible and its inverse reads $\left(\mathcal{P}_{x_{0}}^{x}\right)^{-1}=\mathcal{P}_{x}^{x_{0}}: T_{x, \delta} \mathscr{B}_{t} \rightarrow T_{x_{0}, \delta} \mathscr{B}_{t}$. In addition, $\mathcal{P}_{x_{0}}^{x_{0}}$ is the identity operator from $T_{x_{0}, \delta} \mathscr{B}_{t}$ into itself.

- To represent $\mathfrak{f}_{\alpha}(x, \tilde{x})$ properly, we explain in detail our understanding of the procedure sketched in [106]. For this purpose, we start recalling that $\mathfrak{f}_{\alpha}(x, \tilde{x})$ measures how, at time $t$, the value of $\operatorname{grad} c_{\mathrm{a}}(\tilde{x}, t)$ is "felt" at $x$, for all pairs of points $x, \tilde{x} \in \mathscr{B}_{t}$, such that $\tilde{x} \in \mathscr{U}_{t}(x, \delta)$, with $\delta>0$. This influence has to be described in a way respectful of the geometry of the manifold, which can be achieved as follows. Given $\mathfrak{f}_{\alpha}(x, \tilde{x})$, we select arbitrarily a point $x_{0} \in \mathscr{B}_{t}$ and we introduce an auxiliary function $\mathfrak{f}_{\alpha}^{(0)}\left(x_{0}, \cdot\right): \mathscr{U}_{t}\left(x_{0}, \delta\right) \rightarrow \mathbb{R}$, such that, for an appropriate $\tilde{x}_{0} \in \mathscr{U}_{t}\left(x_{0}, \delta\right), \mathfrak{f}_{\alpha}^{(0)}\left(x_{0}, \tilde{x}_{0}\right)=\mathfrak{f}_{\alpha}(x, \tilde{x})$. In order for $\tilde{x}_{0}$ to be "appropriate", it has to depend on $x$ and $\tilde{x}$ (and on $x_{0}$ ). This can be obtained by calling for the operator

$$
\mathcal{T}_{x}^{x_{0}}:=\exp _{x_{0}} \circ\left(\mathcal{P}_{x_{0}}^{x}\right)^{-1} \circ \exp _{x}^{-1}: \mathscr{U}_{t}(x, \delta) \rightarrow \mathscr{U}_{t}\left(x_{0}, \delta\right) .
$$

As anticipated above, for each $\tilde{x} \in \mathscr{U}_{t}(x, \delta)$, $\exp _{x}^{-1}$ returns a vector $\boldsymbol{v}_{x}$, such that $\left\|\boldsymbol{v}_{x}\right\| \leq \delta$. Then, $\left(\mathcal{P}_{x_{0}}^{x}\right)^{-1}$ transports $\boldsymbol{v}_{x}$ parallely to $x_{0}$, so that $\left(\mathcal{P}_{x_{0}}^{x}\right)^{-1} \boldsymbol{v}_{x}=\boldsymbol{v}_{x_{0}}$. Finally, the operator $\exp _{x_{0}}$ maps $\boldsymbol{v}_{x_{0}}$ into $\tilde{x}_{0}=\exp _{x_{0}}\left(\boldsymbol{v}_{x_{0}}\right) \in \mathscr{U}_{t}\left(x_{0}, \delta\right)$. Therefore, it holds that $\tilde{x}_{0}=\mathcal{T}_{x}^{x_{0}}(\tilde{x})$, thereby explaining how $\tilde{x}_{0}$ depends on $x$ and $\tilde{x}$, for a given $x_{0}$. More specifically, the action of $\mathcal{T}_{x}^{x_{0}}$ on $\tilde{x}$ permits to find the only $\tilde{x}_{0}$ such that Equation $(66)$ becomes

$$
\mathfrak{f}_{\alpha}(x, \tilde{x})=\mathfrak{f}_{\alpha}^{(0)}\left(x_{0}, \mathcal{T}_{x}^{x_{0}}(\tilde{x})\right)=\mathfrak{f}_{\alpha}^{(0)}\left(x_{0}, \tilde{x}_{0}\right),
$$

where the composition $\mathfrak{f}_{\alpha}(x, \cdot)=\mathfrak{f}_{\alpha}^{(0)}\left(x_{0}, \cdot\right) \circ \mathcal{T}_{x}^{x_{0}}: \mathscr{U}_{t}(x, \delta) \rightarrow \mathbb{R}$ is implied. The essence of this result is that the information on the non-locality of a given phenomenon between $x$ and $\tilde{x}$, encompassed by $\mathfrak{f}_{\alpha}(x, \tilde{x})$, is "transported" to the pair of points $x_{0}$ and $\tilde{x}_{0}$ (see Fig. 7).

To conclude, we notice that, in an affine space or, more generally, in a flat subset of an affine space, the procedure outlined above boils down to the determination of the unique point $\tilde{x}_{0}$ such that $\boldsymbol{v}_{x_{0}}=\tilde{x}_{0}-x_{0}$ is equipollent to $\boldsymbol{v}_{x}=\tilde{x}-x$, for given $x_{0}, x$ and $\tilde{x}$. Indeed, within this framework, $\mathcal{T}_{x}^{x_{0}}$ operates in such a way that $\boldsymbol{v}_{x_{0}}=\mathcal{T}_{x}^{x_{0}}(\tilde{x})-x_{0}=\tilde{x}_{0}-x_{0}$ is parallel to $\boldsymbol{v}_{x}$ (because $\boldsymbol{v}_{x}$ is transported parallely along the geodesic - now, a straight line - connecting $x$ with $\left.x_{0}\right)$ and $\left\|\boldsymbol{v}_{x_{0}}\right\| \equiv\left\|\tilde{x}_{0}-x_{0}\right\|=\|\tilde{x}-x\| \equiv\left\|\boldsymbol{v}_{x}\right\|$. Moreover, $\mathfrak{f}_{\alpha}(x, \tilde{x})$ and $\mathfrak{f}_{\alpha}^{(0)}\left(x_{0}, \tilde{x}_{0}\right)$ can be rephrased as $\mathfrak{f}_{\alpha}(x, \tilde{x})=\hat{\mathfrak{f}}_{\alpha}(x-\tilde{x})$ and $\mathfrak{f}_{\alpha}^{(0)}\left(x_{0}, \tilde{x}_{0}\right)=\hat{\mathfrak{f}}_{\alpha}^{(0)}\left(x_{0}-\tilde{x}_{0}\right)$, respectively, and Equation (66), or Equation (71), is trivially satisfied. In this respect, we say that Equation (66) adapts the meaning of convolution from the case of an affine space to the case of a manifold (see Fig. 8). 


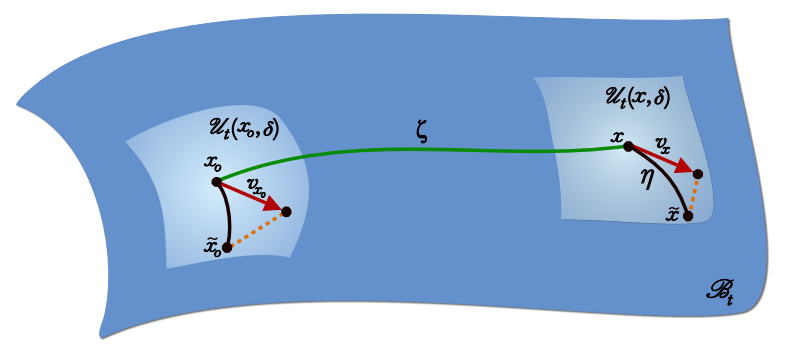

Figure 7: The convolution on manifolds is defined by transporting $\mathfrak{f}_{\alpha}(x, \cdot): \mathscr{U}_{t}(x, \delta) \rightarrow \mathbb{R}$ to every point of $\mathscr{B}_{t}$, while taking into account the manifold geometry. Thus, given a point $\tilde{x}=\eta(1) \in \mathscr{U}_{t}(x, \delta)$, the operation $\exp _{x}^{-1}(\tilde{x})$ returns the vector $\boldsymbol{v}_{x}=\eta^{\prime}(0)$, which is parallelly transported to $\boldsymbol{v}_{x_{0}}$ through a geodesic $\zeta:[0, s] \rightarrow \mathscr{B}_{t}$ connecting $x=\zeta(s)$ and $x_{0}=\zeta(0)$, and the operation $\exp _{x_{0}}\left(\boldsymbol{v}_{x_{0}}\right)$ returns the point $\tilde{x}_{0} \in \mathscr{U}_{t}\left(x_{0}, \delta\right)$. In this way, $\mathfrak{f}_{\alpha}(x, \cdot)$ is transported from $\mathscr{U}_{t}(x, \delta)$ to $\mathscr{U}_{t}\left(x_{0}, \delta\right)$.

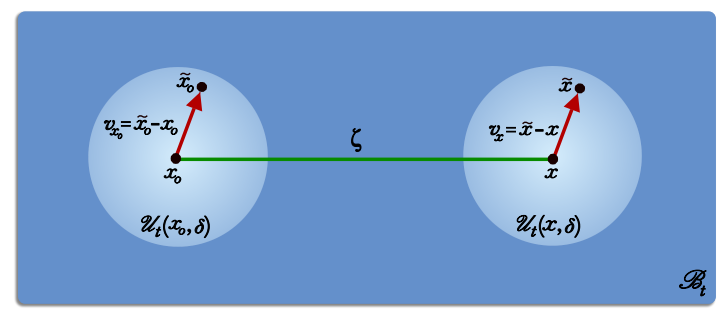

Figure 8: In a flat subset of an affine space $\boldsymbol{v}_{x_{0}}=\tilde{x}_{0}-x_{0}$ is equipollent to $\boldsymbol{v}_{x}=\tilde{x}-x$. Therefore, $\mathfrak{f}_{\alpha}(x, \tilde{x})$ and $\mathfrak{f}_{\alpha}^{(0)}\left(x_{0}, \tilde{x}_{0}\right)$ can be rephrased as $\mathfrak{f}_{\alpha}(x, \tilde{x})=\hat{\mathfrak{f}}_{\alpha}(x-\tilde{x})$ and $\mathfrak{f}_{\alpha}^{(0)}\left(x_{0}, \tilde{x}_{0}\right)=$ $\hat{\mathfrak{f}}_{\alpha}^{(0)}\left(x_{0}-\tilde{x}_{0}\right)$.

\section{References}

[1] Fayçal Ben Adda. La différentiabilité dans le calcul fractionnaire. Comptes Rendus de l'Académie des Sciences - Series I - Mathematics, 326(7):787-791, apr 1998.

[2] Elias C Aifantis and James B Serrin. The mechanical theory of fluid interfaces and maxwell's rule. Journal of Colloid and Interface Science, 96(2):517-529, dec 1983.

[3] Gioacchino Alotta, Mario Di Paola, Francesco Paolo Pinnola, and Massimiliano Zingales. A fractional nonlocal approach to nonlinear blood flow in small-lumen arterial vessels. Meccanica, 55(4):891-906, mar 2020.

[4] D. Ambrosi, G.A. Ateshian, E.M. Arruda, S.C. Cowin, J. Dumais, A. Goriely, G.A. Holzapfel, J.D. Humphrey, R. Kemkemer, E. Kuhl, J.E. Olberding, L.A. Taber, and K. Garikipati. Perspectives on biological growth and remodeling. Journal of the Mechanics and Physics of Solids, 59(4):863-883, apr 2011.

[5] D. Ambrosi and F. Mollica. On the mechanics of a growing tumor. International Journal of Engineering Science, 40(12):1297-1316, jul 2002. 
[6] D. Ambrosi and L. Preziosi. On the closure of mass balance models for tumor growth. Mathematical Models and Methods in Applied Sciences, 12(05):737-754, may 2002.

[7] D. Ambrosi, L. Preziosi, and G. Vitale. The insight of mixtures theory for growth and remodeling. Z. Angew. Math. Phys., 61:177-191, 2010.

[8] R. P. Araujo and D. L. McElwain. A history of the study of solid tumour growth: the contribution of mathematical modelling. Bulletin of Mathematical Biology, may 2004.

[9] T. M. Atanacković, S. Pilipović, B. Stanković, and D. Zorica. Fractional Calculus with Applications in Mechanics: Vibrations and Diffusion Processes. ISTE Ltd., 2014.

[10] T M Atanackovic, S Pilipovic, and D Zorica. A diffusion wave equation with two fractional derivatives of different order. Journal of Physics A: Mathematical and Theoretical, 40(20):5319-5333, apr 2007.

[11] T. M. Atanackovic and B. Stankovic. Generalized wave equation in nonlocal elasticity. Acta Mechanica, 208(1-2):1-10, nov 2008.

[12] Teodor M. Atanacković, S. Pilipović, B. Stanković, and D. Zorica. Fractional Calculus with Applications in Mechanics: Wave Propagation, Impact and Variational Principles. ISTE Ltd., 2014.

[13] G.A. Ateshian and J.A. Weiss. Anisotropic hydraulic permeability under finite deformation. J. Biomech. Engng., 132:111004-1-111004-7, 2010.

[14] N. Bellomo and L. Preziosi. Modelling and mathematical problems related to tumor evolution and its interaction with the immune system. Mathematical and Computer Modelling, 32(34):413-452, aug 2000 .

[15] L.S. Bennethum, M.A. Murad, and J.H. Cushman. Macroscale thermodynamics and the chemical potential for swelling porous media. Transport in Porous Media, 39(2):187-225, 2000 .

[16] Haim Brezis. Functional Analysis, Sobolev Spaces and Partial Differential Equations. Springer New York, 2010.

[17] Michael M. Bronstein, Joan Bruna, Yann LeCun, Arthur Szlam, and Pierre Vandergheynst. Geometric deep learning: Going beyond euclidean data. IEEE Signal Processing Magazine, 34(4):18-42, jul 2017.

[18] Alfonso Bueno-Orovio, David Kay, Vicente Grau, Blanca Rodriguez, and Kevin Burrage. Fractional diffusion models of cardiac electrical propagation: role of structural heterogeneity in dispersion of repolarization. Journal of The Royal Society Interface, 11(97):20140352, aug 2014 .

[19] H. Byrne and L. Preziosi. Modelling solid tumour growth using the theory of mixtures. Mathematical Medicine and Biology, 20(4):341-366, dec 2003. 
[20] H.M. Byrne and M.A. Chaplain. Growth of nonnecrotic tumors in the presence and absence of inhibitors. Mathematical biosciences, 130:151-181, December 1995.

[21] Silvia Capuani, Marco Palombo, Andrea Gabrielli, Augusto Orlandi, Bruno Maraviglia, and Francesco S. Pastore. Spatio-temporal anomalous diffusion imaging: results in controlled phantoms and in excised human meningiomas. Magnetic Resonance Imaging, 31(3):359-365, apr 2013.

[22] A. Carpinteri, P. Cornetti, and A. Sapora. A fractional calculus approach to nonlocal elasticity. The European Physical Journal Special Topics, 193(1):193-204, mar 2011.

[23] J.J. Casciari, S.V. Sotirchos, and R.M. Sutherland. Mathematical modelling of microenvironment and growth in EMT6/ro multicellular tumour spheroids. Cell Proliferation, 25(1):1-22, jan 1992.

[24] Joseph J. Casciari, Stratis V. Sotirchos, and Robert M. Sutherland. Variations in tumor cell growth rates and metabolism with oxygen concentration, glucose concentration, and extracellular pH. Journal of Cellular Physiology, 151(2):386-394, may 1992.

[25] M.A.J. Chaplain, L. Graziano, and L. Preziosi. Mathematical modelling of the loss of tissue compression responsiveness and its role in solid tumour development. Mathematical Medicine and Biology: A Journal of the IMA, 23(3):197-229, sep 2006.

[26] A.S. Chaves. A fractional diffusion equation to describe lévy flights. Physics Letters A, 239(1-2):13-16, feb 1998.

[27] V. Ciancio, M. Dolfin, M. Francaviglia, and S. Preston. Uniform materials and the multiplicative decomposition of the deformation gradient in finite elasto-plasticity. J. Non-Equilib. Thermodyn., 33(3):199-234, 2008.

[28] P. Ciarletta, M. Destrade, and A. L. Gower. On residual stresses and homeostasis: an elastic theory of functional adaptation in living matter. Scientific Reports, 6(1), apr 2016.

[29] D Cioranescu. An introduction to homogenization. Oxford University Press, 1999.

[30] S. Cleja-Tigoiu and G. A. Maugin. Eshelby's stress tensors in finite elastoplasticity. Acta Mechanica, 139(1-4):231-249, mar 2000.

[31] Helena L.E. Coker, Matthew R. Cheetham, Daniel R. Kattnig, Yong J. Wang, Sergi GarciaManyes, and Mark I. Wallace. Controlling anomalous diffusion in lipid membranes. Biophysical Journal, 116(6):1085-1094, mar 2019.

[32] S. C. Cowin and G. A. Holzapfel. On the modeling of growth and adaptation. In Holzapfel G. A. and Odgen R. W., editors, Mechanics of Biological Tissue, pages 29-46. Springer-Verlag, 2006.

[33] E. Crevacore, S. Di Stefano, and A. Grillo. Coupling among deformation, fluid flow, structural reorganisation and fibre reorientation in fibre-reinforced, transversely isotropic biological tissues. International Journal of Nonlinear Mechanics, In press, 2018. 
[34] Y. Danyuo, C. J. Ani, A. A. Salifu, J. D. Obayemi, S. Dozie-Nwachukwu, V. O. Obanawu, U. M. Akpan, O. S. Odusanya, M. Abade-Abugre, F. McBagonluri, and W. O. Soboyejo. Anomalous release kinetics of prodigiosin from poly-n-isopropyl-acrylamid based hydrogels for the treatment of triple negative breast cancer. Scientific Reports, 9(1), mar 2019.

[35] D. del Castillo-Negrete. Fractional diffusion models of nonlocal transport. Physics of Plasmas, 13(8):082308, aug 2006.

[36] Zhi-Qiang Deng, Vijay P. Singh, and Lars Bengtsson. Numerical solution of fractional advection-dispersion equation. Journal of Hydraulic Engineering, 130(5):422-431, may 2004.

[37] Mario Di Paola and Massimiliano Zingales. Long-range cohesive interactions of non-local continuum faced by fractional calculus. International Journal of Solids and Structures, 45(21):5642-5659, oct 2008 .

[38] Mario Di Paola and Massimiliano Zingales. Fractional differential calculus for 3d mechanically based non-local elasticity. International Journal for Multiscale Computational Engineering, 9(5):579-597, 2011.

[39] A. DiCarlo and S. Quiligotti. Growth and balance. Mechanics Research Communications, 29(6):449-456, nov 2002.

[40] Nader Engheta. Fractional curl operator in electromagnetics. Microwave and Optical Technology Letters, 17(2):86-91, feb 1998.

[41] M. Epstein. Self-driven continuous dislocations and growth. In Maugin G.A. Steinmann P., editor, Mechanics of Material Forces. Advances in Mechanics and Mathematics, volume 11, pages 129-139. Springer, Boston, MA, 2005.

[42] M. Epstein and G.A. Maugin. Thermomechanics of volumetric growth in uniform bodies. International Journal of Plasticity, 16(7-8):951-978, jun 2000.

[43] A. Cemal Eringen. Linear theory of nonlocal elasticity and dispersion of plane waves. International Journal of Engineering Science, 10(5):425-435, may 1972.

[44] Gissell Estrada-Rodriguez, Heiko Gimperlein, Kevin J. Painter, and Jakub Stocek. Spacetime fractional diffusion in cell movement models with delay. Mathematical Models and Methods in Applied Sciences, 29(01):65-88, jan 2019.

[45] N. Filipovitch, K. M. Hill, A. Longjas, and V. R. Voller. Infiltration experiments demonstrate an explicit connection between heterogeneity and anomalous diffusion behavior. Water Resources Research, 52(7):5167-5178, jul 2016.

[46] G. Forgacs, R.A. Foty, Y. Shafrir, and M.S. Steinberg. Viscoelastic properties of living embryonic tissues: a quantitative study. Biophysical Journal, 74:2227-2234, 1998.

[47] P. Fuschi, A. A. Pisano, and D. De Domenico. Plane stress problems in nonlocal elasticity: finite element solutions with a strain-difference-based formulation. Journal of Mathematical Analysis and Applications, 431(2):714-736, nov 2015. 
[48] Naama Gal and Daphne Weihs. Experimental evidence of strong anomalous diffusion in living cells. Physical Review E, 81(2), feb 2010.

[49] Heiko Gimperlein and Jakub Stocek. Space-time adaptive finite elements for nonlocal parabolic variational inequalities. Computer Methods in Applied Mechanics and Engineering, 352:137-171, aug 2019.

[50] C. Giverso and L. Preziosi. Modelling the compression and reorganization of cell aggregates. Mathematical Medicine and Biology, 29:181-204, 2012.

[51] C. Giverso, M. Scianna, and A. Grillo. Growing avascular tumours as elasto-plastic bodies by the theory of evolving natural configurations. Mech. Res. Commun., 68:31-39, 2015.

[52] A. Goriely. The Mathematics and Mechanics of Biological Growth. Springer New York, 2016.

[53] A. Grillo, M. Carfagna, and S. Federico. Non-Darcian flow in fibre-reinforced biological tissues. Meccanica, 52:3299-3320, 2017.

[54] A. Grillo, S. Federico, and G. Wittum. Growth, mass transfer, and remodeling in fiberreinforced, multi-constituent materials. Int. J. Nonlinear Mech., 47:388-401, 2012.

[55] A. Grillo, R. Prohl, and G. Wittum. A poroplastic model of structural reorganisation in porous media of biomechanical interest. Continuum Mech. Therm., 28:579-601, 2016.

[56] Alfio Grillo, Salvatore Di Stefano, Ariel Ramírez-Torres, and Michele Loverre. A study of growth and remodeling in isotropic tissues, based on the anand-aslan-chester theory of straingradient plasticity. GAMM-Mitteilungen, 42(4), may 2019.

[57] Morton E. Gurtin. On the plasticity of single crystals: free energy, microforces, plastic-strain gradients. Journal of the Mechanics and Physics of Solids, 48(5):989-1036, may 2000.

[58] Klaus Hackl and Franz Dieter Fischer. On the relation between the principle of maximum dissipation and inelastic evolution given by dissipation potentials. Proceedings of the Royal Society A: Mathematical, Physical and Engineering Sciences, 464(2089):117-132, oct 2007.

[59] Kotaybah Hashlamoun, Ziad Abusara, Ariel Ramírez-Torres, Alfio Grillo, Walter Herzog, and Salvatore Federico. Fluorescence recovery after photobleaching: direct measurement of diffusion anisotropy. Biomechanics and Modeling in Mechanobiology, jun 2020.

[60] S.M. Hassanizadeh. Derivation of basic equations of mass transp. porous med., part 2. generalized darcy's and fick's laws. Adv. Water Resour., 9:207-222, 1986.

[61] Gabriel Helmlinger, Paolo A. Netti, Hera C. Lichtenbeld, Robert J. Melder, and Rakesh K. Jain. Solid stress inhibits the growth of multicellular tumor spheroids. Nature Biotechnology, 15(8):778-783, aug 1997.

[62] M.H. Holmes and V.C. Mow. The nonlinear characteristics of soft gels and hydrated connective tissues in ultrafiltration. Journal of biomechanics, 23:1145-1156, 1990. 
[63] Quanzhong Huang, Guanhua Huang, and Hongbin Zhan. A finite element solution for the fractional advection-dispersion equation. Advances in Water Resources, 31(12):1578-1589, dec 2008 .

[64] J. D. Humphrey. Towards a theory of vascular growth and remodeling. In Holzapfel G.A. and Ogden R.W., editors, Mechanics of Biological Tissue, pages 3-15. Springer-Verlag, 2006.

[65] Ruben Interian, Reinaldo Rodríguez-Ramos, Fernando Valdés-Ravelo, Ariel Ramírez-Torres, Celso Ribeiro, and Aura Conci. Tumor growth modelling by cellular automata. Mathematics and Mechanics of Complex Systems, 5(3-4):239-259, oct 2017.

[66] R.K. Jain, J.D. Martin, and T. Stylianopoulos. The role of mechanical forces in tumor growth and therapy. Annual Review of Biomedical Engineering, 16:321-346, 2014.

[67] Chongming Jiang, Chunyan Cui, Li Li, and Yuanzhi Shao. The anomalous diffusion of a tumor invading with different surrounding tissues. PLoS ONE, 9(10):e109784, oct 2014.

[68] Ansgar Jüngel and Ines Viktoria Stelzer. Entropy structure of a cross-diffusion tumor-growth model. Mathematical Models and Methods in Applied Sciences, 22(07):1250009, may 2012.

[69] M A Konerding, E Fait, and A Gaumann. 3d microvascular architecture of pre-cancerous lesions and invasive carcinomas of the colon. British Journal of Cancer, 84(10):1354-1362, 2001.

[70] M. Köpf, C. Corinth, O. Haferkamp, and T.F. Nonnenmacher. Anomalous diffusion of water in biological tissues. Biophysical Journal, 70(6):2950-2958, jun 1996.

[71] R. Krishna and J.A. Wesselingh. The maxwell-stefan approach to mass transfer. Chemical Engineering Science, 52(6):861-911, mar 1996.

[72] E. Kröner. Elasticity theory of materials with long range cohesive forces. International Journal of Solids and Structures, 3(5):731-742, sep 1967.

[73] Ellen Kuhl. Growing matter: A review of growth in living systems. J. Mech. Behav. Biomed. Mater., 29:529-543, jan 2014.

[74] Daniel J. Lacks. Tortuosity and anomalous diffusion in the neuromuscular junction. Physical Review E, 77(4), apr 2008.

[75] E. K. Lenzi, H. V. Ribeiro, A. A. Tateishi, R. S. Zola, and L. R. Evangelista. Anomalous diffusion and transport in heterogeneous systems separated by a membrane. Proceedings of the Royal Society A: Mathematical, Physical and Engineering Sciences, 472(2195):20160502, nov 2016.

[76] V.E. Lynch, B.A. Carreras, D. del Castillo-Negrete, K.M. Ferreira-Mejias, and H.R. Hicks. Numerical methods for the solution of partial differential equations of fractional order. Journal of Computational Physics, 192(2):406-421, dec 2003.

[77] P. Macklin, Vittorio Cristini, and John Lowengrub. Biological background. In Multiscale Modeling of Cancer, pages 8-23. Cambridge University Press, 2010. 
[78] Paul Macklin, Steven McDougall, Alexander R. A. Anderson, Mark A. J. Chaplain, Vittorio Cristini, and John Lowengrub. Multiscale modelling and nonlinear simulation of vascular tumour growth. Journal of Mathematical Biology, 58(4-5):765-798, sep 2009.

[79] J.E. Marsden and T.J.R. Hughes. Mathematical Foundations of Elasticity. Dover Publications, Inc., Mineola, New York, 1983.

[80] P. Mascheroni, M. Carfagna, A. Grillo, D.P. Boso, and B.A. Schrefler. An avascular tumor growth model based on porous media mechanics and evolving natural states. Mathematics and Mechanics of Solids, 23(4):686-712, jun 2018.

[81] P. Mascheroni, C. Stigliano, M. Carfagna, D.P. Boso, L. Preziosi, P. Decuzzi, and B.A. Schrefler. Predicting the growth of glioblastoma multiforme spheroids using a multiphase porous media model. Biomech. Model. Mechanobiol., 15(5):1215-1228, jan 2016.

[82] Mark M. Meerschaert, Jeff Mortensen, and Stephen W. Wheatcraft. Fractional vector calculus for fractional advection-dispersion. Physica A: Statistical Mechanics and its Applications, $367: 181-190$, jul 2006.

[83] Mark M. Meerschaert and Charles Tadjeran. Finite difference approximations for two-sided space-fractional partial differential equations. Applied Numerical Mathematics, 56(1):80-90, jan 2006 .

[84] Ralf Metzler and Joseph Klafter. The random walk's guide to anomalous diffusion: a fractional dynamics approach. Physics Reports, 339(1):1-77, dec 2000.

[85] M.V. Mićunović. Thermomechanics of Viscoplasticity. Springer New York, 2009.

[86] Shlomo P. Neuman and Daniel M. Tartakovsky. Perspective on theories of non-fickian transport in heterogeneous media. Advances in Water Resources, 32(5):670-680, may 2009.

[87] K. Nishimoto. Fractional Calculus: Integrations and Differentiations of Arbitrary Order. University of New Haven Press, 1989.

[88] Keith B. Oldham and Jerome Spanier. The Fractional Calculus. Theory and Applications of Differentiation and Integration to Arbitrary Order. Elsevier Science, 1974.

[89] P. Paradisi, R. Cesari, F. Mainardi, A. Maurizi, and F. Tampieri. A generalized fick's law to describe non-local transport effects. Physics and Chemistry of the Earth, Part B: Hydrology, Oceans and Atmosphere, 26(4):275-279, jan 2001.

[90] R. Penta and D. Ambrosi. The role of the microvascular tortuosity in tumor transport phenomena. Journal of Theoretical Biology, 364:80-97, jan 2015.

[91] Raimondo Penta, Laura Miller, Alfio Grillo, Ariel Ramírez-Torres, Pietro Mascheroni, and Reinaldo Rodríguez-Ramos. Porosity and diffusion in biological tissues. recent advances and further perspectives. In Constitutive Modelling of Solid Continua, pages 311-356. Springer International Publishing, nov 2020. 
[92] Igor Podlubny. Fractional Differential Equations: An Introduction to Fractional Derivatives, Fractional Differential Equations, to Methods of Their Solution and Some of Their Applications (ISSN Book 198). Academic Press, 1998.

[93] Adrien Poulenard and Maks Ovsjanikov. Multi-directional geodesic neural networks via equivariant convolution. ACM Transactions on Graphics, 37(6):1-14, dec 2018.

[94] S. Preston and M. Elzanowski. Material uniformity and the concept of the stress space. In Bettina Albers, editor, Continuous Media with Microstructure, pages 91-101. Springer-Verlag Berlin Heidelberg, 1 edition, 2010.

[95] L. Preziosi and G. Vitale. A multiphase model of tumor and tissue growth including cell adhesion and plastic reorganization. Math. Models Methods Appl. Sci., 21(09):1901-1932, sep 2011.

[96] Ariel Ramírez-Torres, Salvatore Di Stefano, Alfio Grillo, Reinaldo Rodríguez-Ramos, José Merodio, and Raimondo Penta. An asymptotic homogenization approach to the microstructural evolution of heterogeneous media. International Journal of Non-Linear Mechanics, 106:245-257, nov 2018

[97] Ariel Ramírez-Torres, Reinaldo Rodríguez-Ramos, Rainer Glüge, Julián Bravo-Castillero, Raúl Guinovart-Díaz, and Rocío Rodríguez-Sanchez. Biomechanic approach of a growing tumor. Mechanics Research Communications, 51:32-38, jul 2013.

[98] E.K. Rodriguez, A. Hoger, and A.D. McCulloch. Stress-dependent finite growth in soft elastic tissues. J. Biomech., 27:455-467, 1994.

[99] John Paul Roop. Computational aspects of FEM approximation of fractional advection dispersion equations on bounded domains in $\mathbb{R}^{2}$. Journal of Computational and Applied Mathematics, 193(1):243-268, aug 2006.

[100] Tiina Roose, S. Jonathan Chapman, and Philip K. Maini. Mathematical models of avascular tumor growth. SIAM Review, 49(2):179-208, jan 2007.

[101] S. Di Stefano, A. Ramírez-Torres, R. Penta, and A. Grillo. Self-influenced growth through evolving material inhomogeneities. International Journal of Non-Linear Mechanics, 106:174$187,2018$.

[102] S. Sadik and A. Yavari. On the origins of the idea of the multiplicative decomposition of the deformation gradient. Mathematics and Mechanics of Solids, 22(4):771-772, oct 2017.

[103] Sandro Salsa, Federico M. G. Vegni, Anna Zaretti, and Paolo Zunino. Elementi di analisi funzionale. In UNITEXT, pages 259-324. Springer Milan, 2009.

[104] S. G. Samko, A. A. Kilbas, and O. I. Marichev. Fractional Integrals and Derivatives: Theory and Applications. Gordon and Breach Science Publishers, 1993.

[105] A. Sapora, P. Cornetti, B. Chiaia, E. K. Lenzi, and L. R. Evangelista. Nonlocal diffusion in porous media: A spatial fractional approach. Journal of Engineering Mechanics, 143(5), may 2017. 
[106] Stefan C. Schonsheck, Bin Dong, and Rongjie Lai. Parallel transport convolution: A new tool for convolutional neural networks on manifolds. arXiv preprint, 2018.

[107] G Sciumè, S Shelton, W G Gray, C T Miller, F Hussain, M Ferrari, P Decuzzi, and B A Schrefler. A multiphase model for three-dimensional tumor growth. New J. Phys., 15(1):015005, jan 2013.

[108] Mihir Sen and Eduardo Ramos. A spatially non-local model for flow in porous media. Transport in Porous Media, 92(1):29-39, oct 2011.

[109] S.L. Sobolev. Nonlocal diffusion models: Application to rapid solidification of binary mixtures. International Journal of Heat and Mass Transfer, 71:295-302, apr 2014.

[110] T. Stylianopoulos, J.D. Martin, V.P. Chauhan, S.R. Jain, and et al. Causes, consequences, and remedies for growth-induced solid stress in murine and human tumors. PNAS, 109(38):15101-15108, 2012.

[111] T. Stylianopoulos, J.D. Martin, M. Snuderl, F. Mpekris, S.R. Jain, and R.K. Jain. Coevolution of solid stress and interstitial fluid pressure in tumors during progression: Implications for vascular collapse. Cancer Research, 73(13):3833-3841, apr 2013.

[112] V. E. Tarasov and G. M. Zaslavsky. Fractional dynamics of systems with long-range interaction. Communications in Nonlinear Science and Numerical Simulation, 11(8):885-898, dec 2006 .

[113] Vasily E. Tarasov. Fractional vector calculus and fractional maxwell's equations. Annals of Physics, 323(11):2756-2778, nov 2008.

[114] A. Tomic, A. Grillo, and S. Federico. Poroelastic materials reinforced by statistically oriented fibres - numerical implementation and application to articular cartilage. IMA J. Appl. Math., 79:1027-1059, 2014.

[115] P. R. Wills, D. J. Scott, and D. J. Winzor. Thermodynamics and thermodynamic nonideality. In G. C. K. Roberts, editor, Encyclopedia of Biophysics, pages 2583-2589. Springer, Berlin Heidelberg, 2013. 